\title{
A staggered-grid finite-difference scheme optimized in the time-space domain for modeling scalar-wave propagation in geophysical problems
}

\author{
Sirui Tan ${ }^{\mathrm{a}}$, Lianjie Huang ${ }^{\mathrm{b}, *}$ \\ ${ }^{a}$ Formerly Los Alamos National Laboratory, Geophysics Group, Los Alamos, NM 87545, USA \\ ${ }^{b}$ Los Alamos National Laboratory, Geophysics Group, Los Alamos, NM 87545, USA
}

\begin{abstract}
For modeling scalar-wave propagation in geophysical problems using finite-difference schemes, optimizing the coefficients of the finite-difference operators can reduce numerical dispersion. Most optimized finite-difference schemes for modeling seismicwave propagation suppress only spatial but not temporal dispersion errors. We develop a novel optimized finite-difference scheme for numerical scalar-wave modeling to control dispersion errors not only in space but also in time. Our optimized scheme is based on a new stencil that contains a few more grid points than the standard stencil. We design an objective function for minimizing relative errors of phase velocities of waves propagating in all directions within a given range of wavenumbers. Dispersion analysis and numerical examples demonstrate that our optimized finite-difference scheme is computationally up to 2.5 times faster than the optimized schemes using the standard stencil to achieve the similar modeling accuracy for a given 2D or 3D problem. Compared with the high-order finite-difference scheme using the same new stencil, our optimized scheme reduces 50 percent of the computational cost to achieve the similar modeling accuracy. This new optimized finite-difference scheme is particularly useful for large-scale 3D scalar-wave modeling and inversion.
\end{abstract}

Keywords: Dispersion error, Finite-difference scheme, Finite-difference stencil, Numerical modeling, Optimized scheme, Scalar wave, Wave propagation

\footnotetext{
${ }^{*}$ Corresponding author, Tel.: +1 (505) 665-1108

Email addresses: siruitan@hotmail.com (Sirui Tan), ljh@lanl.gov (Lianjie Huang)
}

Preprint submitted to Journal of Computational Physics 


\section{Introduction}

Finite-difference (FD) schemes have been widely used for simulations of wave propagation [e.g., 1]. High-order FD schemes are particularly attractive for large-scale 3D modeling, because they are able to control numerical dispersion using a larger grid spacing compared with low-order schemes [e.g., 2, 3]. The coefficients of high-order FD operators are usually determined using the Taylor expansion of the truncation error $\varepsilon$ with respect to the grid spacing $h$ such that $\varepsilon=O\left(h^{2 M}\right)$, where $2 M$ is the length of the standard FD operator or stencil [e.g., 4]. Equivalently, one may express the phase-velocity error $\epsilon_{v}$ in terms of the normalized wavenumber " $k h$ " and design the coefficients such that $\epsilon_{v}=O\left((k h)^{2 M}\right)$ [e.g., 5], where $k$ is the wavenumber. Although very high-order accuracy has been achieved in space, second-order time discretization is popular for modeling large-scale wave propagation because of its relatively low requirements of computer memory [e.g., 6]. In the following, we refer the scheme with $2 M^{\text {th }}$-order accuracy in space and second-order accuracy in time as the standard highorder FD scheme.

The numerical solution obtained using a high-order scheme converges rapidly to the true solution when the grid spacing $h$ approaches zero. In other words, high-order schemes are excellent at controlling the phase-velocity error for low wavenumbers. However, for a given $h$, high-order schemes do not always sufficiently suppress the error for large wavenumbers. One remedy is increasing the length of the FD operator $2 M$, leading to high computational costs. When $2 M$ approaches the total number of grid points along one direction, high-order FD schemes essentially become the pseudospectral method $[7,8]$, which is free of numerical dispersion but is computationally expensive.

Holberg [9] introduced an optimized FD scheme to control spatial dispersion errors for a wide range of wavenumbers for a given length of the FD operator for numerical modeling of seismic-wave propagation. His objective function for optimization is the maximum relative error of the group velocity. Various optimized FD schemes have 
emerged since Holberg's pioneering work.

Numerous optimized FD schemes minimize the spatial dispersion error and temporal dispersion error independently [e.g., 10-14]. Lele [10] obtained compact FD schemes with spectral-like resolution by imposing the requirement that the discrete FD operator matches the spatial differential operator at three prescribed high wavenumbers. Tam and Webb [11] constructed a dispersion-relation-preserving (DRP) scheme by optimizing the FD approximations to the spatial and temporal differential operators. Bogey and Bailly [12] advanced the DRP scheme using spatial FD operators with a length of up to 13 grid points. Hu et al. [13] developed low-dissipation and low-dispersion Runge-Kutta time-advancing schemes. Zhang and Yao [14] found that the norm of objective functions plays an important role for designing optimized FD schemes. Their schemes based on the maximum norm have more flexibility and better accuracy than those in $[11,12]$ based on $L^{2}$ - or $L^{1}$-norm.

Several optimized FD schemes minimize the spatial dispersion error and temporal dispersion error simultaneously. Haras and Ta'asan [15] minimized the global truncation error of the partial differential equation, and demonstrated that their compact FD scheme is more accurate than Lele's spectral-like scheme in [10] for solving the scalarwave equation. Etgen [16] developed an FD scheme to minimize the phase-velocity error. His scheme balances both spatial and temporal dispersion errors because the two types of errors have opposite signs. Stork [17] designed spatial FD operators that vary between consecutive time steps to reduce dispersion errors. Liu [18] found that minimizing the relative error of the time-space-domain dispersion relation can lead to smaller relative errors of the phase velocity, compared to minimizing the absolute error of the space-domain dispersion relation. He obtained his globally optimal FD schemes by linearizing an objective function of the relative phase-velocity error and solving it using a least-squares approach.

The FD schemes optimized in time-space domain in $[9,16-18]$ are based on the standard finite-difference stencil composed of grid points on the axis along which the spatial derivative is calculated. Liu and Sen [5] demonstrated that high-order FD schemes based on the standard $2 M$-point stencil can reach the $2 M^{\text {th }}$-order accuracy both in space and time, but only along eight directions of wave propagation in $2 \mathrm{D}$ and 
48 directions in 3D when using wavefields at one time step for temporal evolution. The temporal accuracy is still second order along the other propagation directions. As high-order FD schemes, optimized schemes based on the standard stencil still have low temporal accuracy when using wavefields at one time step for temporal evolution, and a small time interval has to be used to adequately control temporal dispersion errors.

We recently developed a new staggered-grid finite-difference method in the timespace domain to improve the accuracy in time [19]. The stencil, same as that for LaxWendroff scheme [e.g., 2, 20, 21], contains a few additional grid points off each axis compared to the standard stencil. Our new FD scheme increases the temporal accuracy from second order to fourth order for FD modeling with high-order spatial accuracy. The computer-memory requirement of our FD scheme is similar to that of the standard high-order FD scheme with second-order accuracy in time.

In this paper, we develop an optimized staggered-grid finite-difference scheme in the time-space domain based on our new stencil for solving 2D and 3D scalar-wave equations. Scalar-wave equations are widely used in important geophysical problems, including reverse-time migration [22] and full-waveform inversion [23]. In such problems, the phase error is one of the major concerns. Our objective function for optimization is thus the relative error of the phase velocity for waves propagating in all directions within a given range of wavenumbers. Our optimized scheme not only suppresses spacial dispersion errors for large wavenumbers, but also allows us to use a large time interval and well control time dispersion errors. The advantage of using a large time interval for numerical wave modeling highlights the novelty of our FD scheme. We preform dispersion analysis for our optimized FD scheme, and use our optimized scheme to conduct numerical modeling of scalar-wave propagation in 2D and $3 \mathrm{D}$ complex media. Our results demonstrate that the computational efficiency of our optimized FD scheme is up to 2.5 times higher than that of the optimized schemes based on the standard stencil for a given 2D or 3D modeling problem. Compared with the high-order FD scheme based on the same new stencil, our optimized scheme achieves the same modeling accuracy with only a half of the original computational cost.

We design our optimized FD scheme specifically for modeling scalar-wave propa- 
gation in geophysical problems, as in [9, 14, 16-18]. The development of the optimized FD schemes in [10-13, 15] is motivated by computational fluid dynamics (CFD) problems. Although the two types of problems are both governed by hyperbolic equations, they have their own computational challenges for practical applications. For example, the spectrum content of the waves and propagation distances may be different. Moreover, different boundary conditions are imposed for geophysical problems and CFD problems. For geophysical problems, two types of boundary conditions are usually imposed for the scalar-wave equation: absorbing boundary conditions and free-surface boundary conditions [e.g., 1, 24]. The former is used to truncate an unbounded domain (the Earth) into a bounded one. There have been extensive studies of absorbing boundary conditions for modeling seismic-wave propagation [e.g., 25-27]. Freesurface boundary conditions are prescribed at the air/ocean or air/land interface. For planar free surfaces, the mirror technique [e.g., 1] can be employed to convert the halfspace problem to a whole-space problem because of symmetry. For CFD problems, properly imposing numerical boundary conditions may become very challenging. Addressing the challenge of boundary conditions for CFD problems is out of the scope of this paper.

This paper is organized as follows. We first derive our optimized FD scheme based on our new stencil, followed by stability and dispersion analyses. We then conduct numerical modeling of scalar-wave propagation in 2D and 3D complex media, and compare the accuracy and efficiency of our FD modelings with those obtained using a high-order FD scheme or an FD scheme optimized in the space domain.

\section{An optimized finite-difference scheme in the time-space domain using a new stencil}

The scalar-wave equation is given by

$$
\frac{\partial^{2} p}{\partial t^{2}}-c(\mathbf{x}) \nabla^{2} p=0
$$

where $c(\mathbf{x})$ is the wave speed, $p(\mathbf{x}, t)$ is the pressure, and $\mathbf{x}$ is the spatial position in 2D or $3 \mathrm{D}$. The scalar-wave equation (1) is mathematically equivalent to the velocity-stress 
formulation given by

$$
\begin{aligned}
\frac{\partial p}{\partial t}+K(\mathbf{x}) \nabla \cdot \mathbf{v} & =0 \\
\frac{\partial \mathbf{v}}{\partial t}+\frac{1}{\rho} \nabla p & =0
\end{aligned}
$$

where $\rho$ is the constant density, $K(\mathbf{x})$ is the bulk modulus, and $\mathbf{v}(\mathbf{x}, t)=(u(\mathbf{x}, t), v(\mathbf{x}, t), w(\mathbf{x}, t))^{T}$ is the vector of the velocity wavefield. Wave speed $c(\mathbf{x})$ is related to bulk modulus $K(\mathbf{x})$ and density $\rho$ via $c=\sqrt{K / \rho}$. The velocity-stress formulation (2) is widely used [e.g., 1, 28-30], because it explicitly gives both the pressure and velocity wavefields. We aim to design an optimized staggered-grid FD scheme for solving the first-order system (2).

In the $2 \mathrm{D}$ case, the staggered-grid FD discretization of eq. (2) is given by

$$
\begin{aligned}
p_{0,0}^{j} & =p_{0,0}^{j-1}-K\left(D_{x}^{O} u_{0,0}^{j-1 / 2}+D_{z}^{O} w_{0,0}^{j-1 / 2}\right) \Delta t, \\
u_{1 / 2,0}^{j+1 / 2} & =u_{1 / 2,0}^{j-1 / 2}-\frac{1}{\rho} D_{x}^{O} p_{1 / 2,0}^{j} \Delta t, \\
w_{0,1 / 2}^{j+1 / 2} & =w_{0,1 / 2}^{j-1 / 2}-\frac{1}{\rho} D_{z}^{O} p_{0,1 / 2}^{j} \Delta t,
\end{aligned}
$$

where $\Delta t$ is the time interval. $D_{x}^{O}$ and $D_{z}^{O}$ are optimized finite-difference operators along the $x$ - and $z$-axis, respectively. Term $p_{m, n}^{j}$ represents the discretized pressure wavefield $p(x+m h, z+n h, j \Delta t)$, and $u_{m, n}^{j+1 / 2}$ and $w_{m, n}^{j+1 / 2}$ denote the discretized velocity wavefields. In fact, FD scheme (3) can be written in a form for solving the second-order equation (1) by eliminating discretized velocity wavefields [e.g., 5].

We first briefly review our recently developed high-order FD scheme based on a new stencil [19]. This new FD scheme has the $2 M^{\text {th }}$-order accuracy in space and the fourth-order accuracy in time. We then construct our objective function for optimization. This is a nonlinear optimization problem. We derive an approximate minimizer for numerical optimization of our objective function using the Levenberg-Marquardt algorithm [31]. At the end of this section, we discuss the implementation of absorbing boundary conditions.

\subsection{A new finite-difference stencil}

Our FD scheme uses FD stencils shown in Fig. 1 when $2 M=8$. For the $2 \mathrm{D}$ case, the stencil in Fig. 1a contains $2 M$ grid points on the axis along which the spatial derivative 
is calculated. Four additional grid points lying off the axis play an important role in increasing the temporal accuracy. The FD stencil in the 3D case (Fig. 1b) comprises a stencil in the 2D case and four grid points in the perpendicular plane. Note that the FD stencils in Fig. 1 are also used in Lax-Wendroff scheme [e.g., 2, 20, 21].

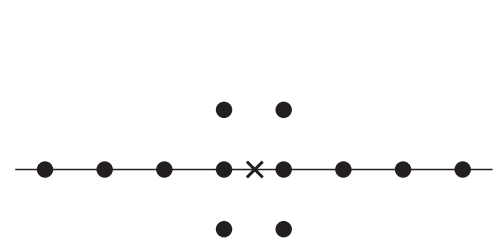

(a) 2D stencil

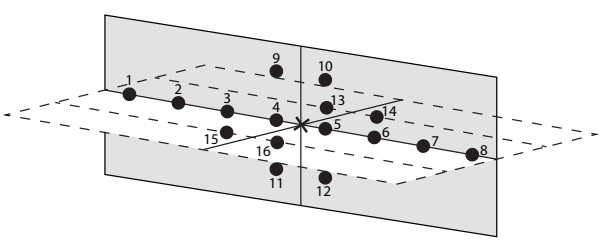

(b) 3D stencil

Figure 1: Illustration of the new FD stencils with $2 M=8$ grid points on the axis for (a) 2D and (b) 3D modeling. Grid points \#1-12 in the shaded plane in (b) are the same as those in the 2D case in (a), while grid points \#13-16 in the perpendicular plane are added for 3D modeling.

The high-order FD operator for 2D modeling is defined on the new stencil as follows

$$
\begin{aligned}
\frac{\partial p}{\partial x} \approx D_{x}^{2 M, 4} p_{0,0}= & \frac{1}{h}\left[\sum_{m=1}^{M} a_{m, 0}\left(p_{m-1 / 2,0}-p_{-m+1 / 2,0}\right)\right. \\
& \left.+a_{1,1}\left(p_{1 / 2,1}-p_{-1 / 2,1}+p_{1 / 2,-1}-p_{-1 / 2,-1}\right)\right],
\end{aligned}
$$

where $h$ denotes the spatial grid spacing, and we omit the superscripts for time steps. The FD operator $D_{x}^{2 M, 4}$ has the $2 M^{\text {th }}$-order accuracy in space and the fourth-order accuracy in time. The finite-difference coefficients in eq. (4) are given by

$$
\begin{aligned}
& a_{1,1}=\frac{r^{2}}{24}, \\
& a_{1,0}=1-2 a_{1,1}-\sum_{m=2}^{M}(2 m-1) a_{m, 0} \\
& a_{m, 0}=\frac{(-1)^{m+1}}{2 m-1} \prod_{l=1, l \neq m}^{M} \frac{(2 l-1)^{2}-r^{2}}{\left|(2 m-1)^{2}-(2 l-1)^{2}\right|}, \quad m=2,3, \cdots, M,
\end{aligned}
$$

where

$$
r=c \Delta t / h
$$


is the Courant-Friedrichs-Lewy (CFL) number. In a heterogeneous medium, $c$ is a function of the spatial position, and can vary from one grid point to another grid point.

For 3D modeling, the high-order FD operator is calculated using

$$
\begin{aligned}
\frac{\partial p}{\partial x} \approx & D_{x}^{2 M, 4} p_{0,0,0}=\frac{1}{h} \sum_{m=1}^{M} a_{m, 0,0}\left(p_{m-1 / 2,0,0}-p_{-m+1 / 2,0,0}\right)+ \\
& \frac{1}{h} a_{1,1,0}\left(p_{1 / 2,1,0}-p_{-1 / 2,1,0}+p_{1 / 2,-1,0}-p_{-1 / 2,-1,0}\right. \\
& \left.+p_{1 / 2,0,1}-p_{-1 / 2,0,1}+p_{1 / 2,0,-1}-p_{-1 / 2,0,-1}\right),
\end{aligned}
$$

where $p_{m, n, l}=p(x+m h, y+n h, z+l h)$ and thus $p_{0,0,0}=p(x, y, z)$. The coefficients in eq. (6) are

$$
\begin{aligned}
& a_{1,1,0}=\frac{r^{2}}{24}, \\
& a_{1,0,0}=1-4 a_{1,1,0}-\sum_{m=2}^{M}(2 m-1) a_{m, 0,0}, \\
& a_{m, 0,0}=\frac{(-1)^{m+1}}{2 m-1} \prod_{l=1, l \neq m}^{M} \frac{(2 l-1)^{2}-r^{2}}{\left|(2 m-1)^{2}-(2 l-1)^{2}\right|}, \quad m=2,3, \cdots, M .
\end{aligned}
$$

The FD coefficients in eqs. (5) and (7) depend on the CFL number $r$ and thus on the local wave speed $c$ for a given grid spacing $h$ and time interval $\Delta t$. This makes the fourth-order accuracy in time possible even only using wavefields at one time step for temporal wavefield evolution.

\subsection{Objective function for optimization}

We define our optimized FD operators on the new stencil in Fig. 1a using

$$
\begin{aligned}
\frac{\partial p}{\partial x} \approx D_{x}^{O} p_{0,0}= & \frac{1}{h}\left[\sum_{m=1}^{M} d_{m, 0}\left(p_{m-1 / 2,0}-p_{-m+1 / 2,0}\right)\right. \\
& \left.+d_{1,1}\left(p_{1 / 2,1}-p_{-1 / 2,1}+p_{1 / 2,-1}-p_{-1 / 2,-1}\right)\right], \\
\frac{\partial p}{\partial z} \approx D_{z}^{O} p_{0,0}= & \frac{1}{h}\left[\sum_{m=1}^{M} d_{m, 0}\left(p_{0, m-1 / 2}-p_{0,-m+1 / 2}\right)\right. \\
& \left.+d_{1,1}\left(p_{1,1 / 2}-p_{1,-1 / 2}+p_{-1,1 / 2}-p_{-1,-1 / 2}\right)\right] .
\end{aligned}
$$


We minimize the relative error of the phase velocity, which is directly related to numerical dispersion. We consider a pressure plane wave $p$ given by

$$
p(\mathbf{x}, t)=\exp \left[i\left(\mathbf{k} \cdot \mathbf{x}-k c_{n u m} t\right)\right],
$$

where $c_{\text {num }}$ is the numerical wave speed and the wavenumber $k=|\mathbf{k}|$. For the FD scheme (3), the relative error of the phase velocity for the plane wave propagating along angle $\theta$ is

$$
e_{2}(k h, \theta, r)=\frac{c_{n u m}}{c}-1=\frac{1}{r k h} \arccos \left(1-2 g_{2} r^{2}\right)-1,
$$

where

$$
\begin{aligned}
g_{2}= & {\left[\sum_{m=1}^{M} d_{m, 0} \sin \left((m-1 / 2) k_{x} h\right)+2 d_{1,1} \sin \left(k_{x} h / 2\right) \cos \left(k_{z} h\right)\right]^{2} } \\
& +\left[\sum_{m=1}^{M} d_{m, 0} \sin \left((m-1 / 2) k_{z} h\right)+2 d_{1,1} \sin \left(k_{z} h / 2\right) \cos \left(k_{x} h\right)\right]^{2},
\end{aligned}
$$

and $\mathbf{k}=\left(k_{x}, k_{z}\right)=k(\cos \theta, \sin \theta)$.

For 3D modeling, our optimized FD operator is defined on the new stencil shown in Fig. 1b using

$$
\begin{aligned}
\frac{\partial p}{\partial x} \approx & D_{x}^{O} p_{0,0,0}=\frac{1}{h} \sum_{m=1}^{M} d_{m, 0,0}\left(p_{m-1 / 2,0,0}-p_{-m+1 / 2,0,0}\right)+\frac{1}{h} d_{1,1,0}\left(p_{1 / 2,1,0}\right. \\
& -p_{-1 / 2,1,0}+p_{1 / 2,-1,0}-p_{-1 / 2,-1,0}+p_{1 / 2,0,1}-p_{-1 / 2,0,1} \\
& \left.+p_{1 / 2,0,-1}-p_{-1 / 2,0,-1}\right) .
\end{aligned}
$$

The definitions of $D_{y}^{O}$ and $D_{z}^{O}$ are similar. The relative error of the phase velocity for the plane wave $p(\mathbf{x}, t)=\exp \left[i\left(\mathbf{k} \cdot \mathbf{x}-k c_{n u m} t\right)\right]$ propagating along a direction with angles $(\theta, \phi)$ is given by

$$
e_{3}(k h, \theta, \phi, r)=\frac{c_{\text {num }}}{c}-1=\frac{1}{r k h} \arccos \left(1-2 g_{3} r^{2}\right)-1,
$$


where

$$
\begin{aligned}
g_{3}= & {\left[\sum_{m=1}^{M} d_{m, 0,0} \sin \left((m-1 / 2) k_{x} h\right)+2 d_{1,1,0} \sin \left(k_{x} h / 2\right)\left(\cos \left(k_{y} h\right)+\cos \left(k_{z} h\right)\right)\right]^{2} } \\
& +\left[\sum_{m=1}^{M} d_{m, 0,0} \sin \left((m-1 / 2) k_{y} h\right)+2 d_{1,1,0} \sin \left(k_{y} h / 2\right)\left(\cos \left(k_{x} h\right)+\cos \left(k_{z} h\right)\right)\right]^{2} \\
& +\left[\sum_{m=1}^{M} d_{m, 0,0} \sin \left((m-1 / 2) k_{z} h\right)+2 d_{1,1,0} \sin \left(k_{z} h / 2\right)\left(\cos \left(k_{y} h\right)+\cos \left(k_{x} h\right)\right)\right]^{2},
\end{aligned}
$$

and $\mathbf{k}=\left(k_{x}, k_{y}, k_{z}\right)=k(\sin \theta \cos \phi, \sin \theta \sin \phi, \cos \theta)$.

Our objective function accounts for waves propagating along all directions within a given range of wavenumbers. For 2D modeling, we construct the objective function as

$$
E_{2}(r)=\int_{0}^{\pi / 4} \int_{0}^{q \pi} e_{2}(k h, \theta, r)^{2} d(k h) d \theta
$$

Here the wavenumber range is chosen as $[0, q \pi]$, where $0<q \leq 1$. We only consider the propagation angle in the range of $[0, \pi / 4]$ because of symmetry. Similarly, our objective function for 3D modeling is constructed as

$$
E_{3}(r)=\int_{0}^{\pi / 4} \int_{0}^{\pi / 4} \int_{0}^{q \pi} e_{3}(k h, \theta, \phi, r)^{2} d(k h) d \theta d \phi .
$$

We preform optimization within the wavenumber range $[0, q \pi]$. The larger the value of $q$ is, the wider is the range of wavenumbers under optimization, but less accurate are modeling results for low wavenumbers. Our optimized FD scheme trades off between the relative error of the phase velocity for low wavenumbers and that for large wavenumbers.

\subsection{Solving the nonlinear least-squares problems}

For a given CFL number $r$, our objective functions (13) and (14) lead to nonlinear least-squares problems for the FD coefficients. To avoid nonlinearity, Liu [18] obtained his conventional-grid FD scheme by minimizing the relative error of the dispersion relation. He claimed that the solution is almost the same as that obtained by minimizing the relative error of the phase velocity. However, a direct application of his technique is not able to completely remove the nonlinearity in our problems, because $g_{2}$ and $g_{3}$ 
in eqs. (10) and (12) are nonlinear with respect to the FD coefficients for staggeredgrid FD schemes. We have to use some numerical techniques to solve our nonlinear problems.

We discretize our objective functions using quadratures:

$$
\begin{aligned}
& E_{2}(r) \approx \frac{q \pi^{2}}{4} \frac{1}{N_{1}} \frac{1}{N_{2}} \sum_{m=1}^{N_{1}} \sum_{n=1}^{N_{2}} e_{2}\left(k h_{m}, \theta_{n}, r\right)^{2}, \\
& E_{3}(r) \approx \frac{q \pi^{3}}{16} \frac{1}{N_{1}} \frac{1}{N_{2}^{2}} \sum_{m=1}^{N_{1}} \sum_{n=1}^{N_{2}} \sum_{l=1}^{N_{2}} e_{3}\left(k h_{m}, \theta_{n}, \phi_{l}, r\right)^{2},
\end{aligned}
$$

where $k h_{m}$ are $N_{1}$ uniform samples of the normalized wavenumbers in the range of $[0, q \pi]$, and $\theta_{n}, \phi_{l}$ are $N_{2}$ uniform samples of the propagation angles in the range of $[0, \pi / 4]$.

We employ the Levenberg-Marquardt algorithm [31] to solve the discrete nonlinear least-squares problems (15) using the coefficients of the high-order FD scheme in eqs. (5) and (7) as the initial guesses. We find that the relative error of the phase velocity obtained using $N_{1}=50$ and $N_{2}=2$ is almost the same as that obtained with $N_{1}=50$ and $N_{2}=50$. This means that the phase errors of waves propagating along the axes and the diagonal directions are the most important ones to be minimized.

Note that eq. (15) depends on the CFL number $r=c(\mathbf{x}) \Delta t / h$ that varies spatially in a heterogeneous medium. To avoid minimizing eq. (15) for $r$ at each spatial grid point, we assume the coefficients of the optimized FD schemes are polynomials of degree $M-1$ in $r$. The polynomials are derived by minimizing eq. (15) for $2 M$ uniform samples of $r$ and performing a least-squares fitting of these samples.

In our implementation, we pre-compute and store the values of the FD coefficients for the wave-speed values in a given model. For example, for a given model with the wave speed ranging from $1500 \mathrm{~m} / \mathrm{s}$ to $5000 \mathrm{~m} / \mathrm{s}$, we calculate and store the FD coefficients for wave speeds of $1500 \mathrm{~m} / \mathrm{s}, 1501 \mathrm{~m} / \mathrm{s}, \cdots, 5000 \mathrm{~m} / \mathrm{s}$ with an increment of $1 \mathrm{~m} / \mathrm{s}$. Less than $1 \mathrm{MB}$ of computer memory is needed to store the values of the FD coefficients using our optimized FD scheme. Therefore, our optimized scheme requires almost the same amount of computer memory as the standard high-order FD scheme for large-scale 3D modeling.

The optimization strategy of our new FD scheme for scalar-wave propagation can 
be employed to improve the computational efficiency of FD modeling of acoustic-wave and elastic-wave propagation.

Hereafter, we focus on the optimized FD scheme using $2 M=16$ grid points along the axis to approximate each spatial derivative in the scalar-wave equation (2). The coefficients of our optimized FD scheme for 2D and 3D modeling problems are provided in Appendix A.

\subsection{Boundary conditions}

In geophysical applications, the Earth is usually considered as an unbounded domain. Absorbing boundary conditions are imposed at boundaries of a computational domain to truncate the unbounded domain into a bounded one. Various absorbing boundary conditions have been developed, e.g., the methods of Clayton and Engquist [25], Engquist and Majda [32], and Higdon [33], the perfectly matched layer (PML) method [34], and the hybrid method of Liu and Sen [27] combining one- and two-way wave equations. Absorbing boundary conditions work properly for FD schemes with very long operators for modeling seismic-wave propagation [e.g., 14, 18, 35].

In all our numerical examples, we employ the unsplit convolutional PML method [26] to effectively eliminate unwanted artificial reflections from all boundaries. Absorbing layers with thickness of 15 grid points are added to the exterior of each boundary. In the absorbing layers, we modify the spatial derivatives in the scalar-wave equation (2). For example, we replace the spatial derivative $\frac{\partial}{\partial x}$ with

$$
\frac{\partial}{\partial \hat{x}}=\frac{1}{\kappa_{x}} \frac{\partial}{\partial x}+\psi_{x},
$$

where $\kappa_{x}$ is a parameter, and $\psi_{x}$ is an auxiliary wavefield defined only in PMLs. On the external edges of PMLs, we use zero ghost values to impose Dirichlet boundary conditions $p=0$ and $\mathbf{v}=0$ for all time $t$. There is thus no need to adjust the stencil or coefficients of FD operators for computing $\frac{\partial}{\partial x}$ in eq. (16). The extra computer-memory requirement of PML is very small for large-scale 3D modeling of seismic-wave propagation where each spatial dimension typically has grid points in the order of 1000 . For example, if we consider a 3D model of $1000^{3}$ grid points, storing wavefields $p$ and $\mathbf{v}$ requires 14.9 GB of computer memory. Storing the wavefields in PMLs only 
needs extra memory of $0.69 \mathrm{~GB}$, which is less than $5 \%$ of the total computer memory required. According to our numerical experiments, the stability condition of our optimized scheme, analyzed in the following section, remains valid for PML. The implementation strategy, computational cost, computer-memory usage, and stability of the unsplit convolutional PML method are discussed in detail in [26].

There have been significant efforts to theoretically prove the numerical stability of various PML methods in the past decade [e.g., 36-39]. Abarbanel et al. [37] found that the long-time stability seems to be strongly affected by the type of the layer and the type of discretization, in particular, the discretization in space. Very recently, Duru [39] found that simply replacing the derivative in eq. (16) with FD operators would result in instability. He employed the simultaneous approximation term (SAT) methodology to weakly impose boundary conditions on the external edges of PMLs and showed that the resulting discrete models do not support growing modes. Duru [39] also found that the interior FD scheme plays little role in numerical stability once the numerical implementation of boundary conditions for the PML is carefully designed. His interior FD scheme is based on summation-by-parts FD operators on standard grids. It is our future research to investigate whether the SAT technique combined with our optimized FD operator on staggered-grids would allow the theoretical proof of numerical stability of PMLs.

At the air/ocean or air/land interface, free-surface boundary condition $p=0$ is sometimes prescribed. For planar free surfaces, the mirror technique [e.g., 1] may be employed, where extra rows of ghost points are added in the air. All interior wavefields are imaged symmetrically or anti-symmetrically to ghost wavefields. The stability condition of the FD scheme for solving the half-space problem is the same as that for solving the whole-space problem because of symmetry.

\section{Analyses of the optimized FD scheme}

We perform stability and dispersion analyses of our optimized FD scheme, and compare the results with those of the high-order scheme using the same FD stencil and with those of the optimized schemes using the standard stencil. 


\subsection{Stability analysis}

For 2D modeling, the standard von Neumann analysis gives

$$
r \leq s(r)=\left[\sqrt{2} \sum_{m=1}^{M}(-1)^{m+1} d_{m, 0}(r)-2 \sqrt{2} d_{1,1}(r)\right]^{-1} .
$$

This stability factor $s$ depends on CFL number $r$. The maximum allowed value of $s$ is determined by the minimum $x$-intercept between the curve $s(r)$ and the straight line $s=r$, as shown in Fig. 2a. This gives the stability condition of our optimized FD scheme for 2D modeling: $r \leq 0.533$. It is slightly more stringent than the stability condition $r \leq 0.607$ for the high-order scheme using the same FD stencil [19].

For 3D modeling, the same stability analysis gives

$$
r \leq s(r)=\left[\sqrt{3} \sum_{m=1}^{M}(-1)^{m+1} d_{m, 0,0}(r)-4 \sqrt{3} d_{1,1,0}(r)\right]^{-1} .
$$

According to Fig. 2b, the stability condition for 3D modeling is $r \leq 0.437$. It is again slightly more stringent than the stability condition $r \leq 0.480$ for the high-order scheme using the same FD stencil [19].

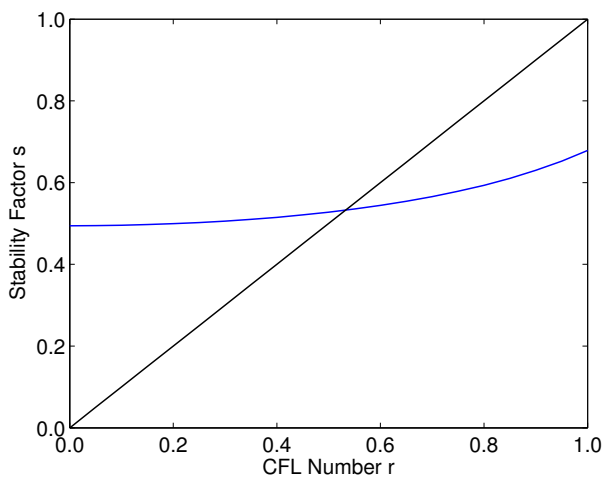

(a) $2 \mathrm{D}$ modeling

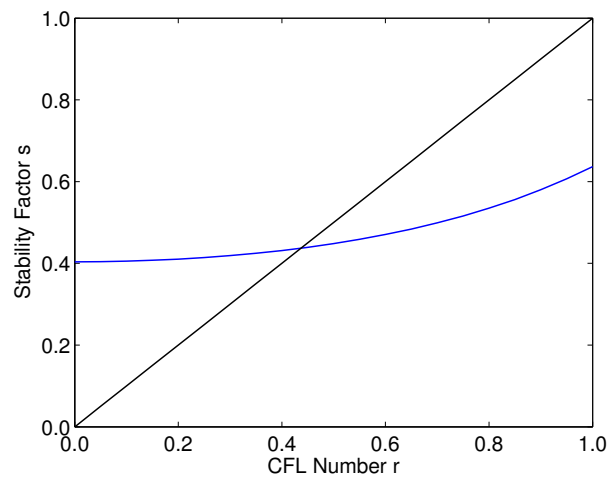

(b) 3D modeling

Figure 2: The relationship between the stability factor $s$ and the CFL number $r$ of our optimized FD scheme for (a) 2D and (b) 3D modeling. The maximum allowed value of $s$ is determined by the minimum $x$-intercept between the curve $s(r)$ (blue curve) and the straight line $s=r$ (black line). 


\subsection{Dispersion analysis}

We calculate the relative error of the phase velocity using eq. (9) for our optimized 2D FD scheme, and display the resulting dispersion curves for propagation angles $\theta=$ $0, \pi / 4$ in Fig. 3. For comparison with an optimized FD scheme obtained using Etgen's approach [16], we solve the same least-squares problem (13) with $d_{1,1}=0$. This FD scheme essentially uses the standard stencil. Both optimized schemes perform well when we use a small time interval with $r=0.05$ (Fig. 3a). Here we choose $r=0.05$ to control the relative error of phase velocity within approximately $0.1 \%$. Note that even stricter phase error thresholds are used in [17] $(0.05 \%)$ and [40] $(0.01 \%)$. When we increase the time interval such that $r=0.15$, the optimized scheme using our new stencil maintains its accuracy for a wide range of wavenumbers, while the optimized scheme based on the standard stencil leads to significant errors even for relatively low wavenumbers (Fig. 3b). This demonstrates that the new FD stencil is crucial for improving the temporal accuracy of numerical wave modeling.

Etgen [16] observed similar errors as shown by the red curves in Fig. $3 b$ for his optimized FD scheme based on the standard stencil. He reduced the errors by requiring the dispersion curves of his scheme match those of a very high-order FD scheme and adding a weight in the objective function. His strategy requires tuning parameters, while our approach directly gives an optimized FD scheme with accurate phase velocities for a wide range of wavenumbers.

We next compare our optimized FD scheme with its high-order counterpart with the same value of $M=8$ for 2D modeling, and depict the dispersion curves in Fig. 4. Our optimized FD scheme significantly extends the range of wavenumbers with accurate phase velocities compared to the high-order FD counterpart, even using the large time interval with $r=0.15$.

Also illustrated in Fig. 4 are the dispersion curves for the optimized 2D FD scheme in the space domain obtained using the objective function of Tam [41]:

$$
E_{\text {space }}=\int_{0}^{q \pi}\left[\sum_{m=1}^{M} 2 d_{m, 0} \sin ((m-1 / 2) k h)-k h\right]^{2} d(k h) .
$$

Compared with our optimized FD scheme, the space-domain optimized FD scheme 


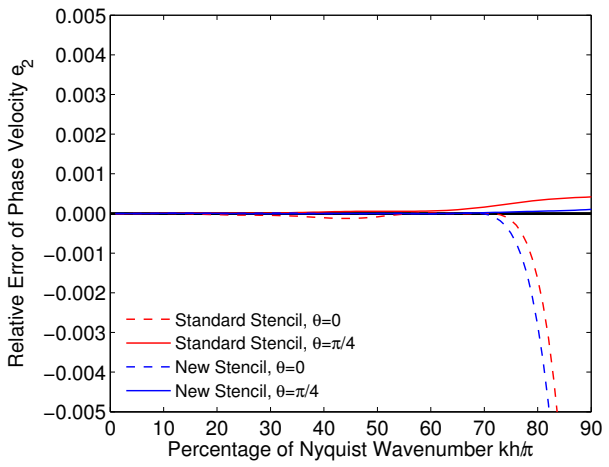

(a) $r=0.05$

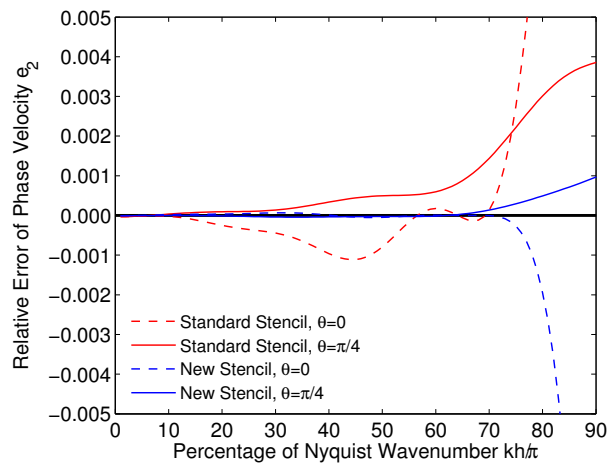

(b) $r=0.15$

Figure 3: Dispersion relations of our optimized FD scheme using the new stencil (blue curves) and the optimized FD scheme using the standard stencil (red curves) for 2D modeling problems with (a) $r=0.05$ and (b) $r=0.15$. Both optimized schemes have good performance when the time interval is small in (a). Only our optimized FD scheme is able to suppress the time dispersion errors using the large time interval in (b). 
based on the objective function (17) gives similar dispersion curves for a small time interval with $r=0.05$ (Fig. 4a). However, a large time interval with $r=0.15$ generates significant time dispersion errors for the space-domain optimized FD scheme, as shown in Fig. 4b. By contrast, our new FD scheme optimized in time-space domain suppresses the errors for a wide range of wavenumbers. Our optimized 3D FD scheme has the same improvement compared with the other FD schemes, as shown in Fig. 5.

We evaluate the computational costs of different FD stencils for one time step using eqs. (8) and (11). The FD schemes using the new stencil with operator length $2 M$ along the axis need $3 M+5$ and $3 M+9$ floating-point operations to compute one spatial derivative for 2D and 3D modeling, respectively, while FD schemes using the standard

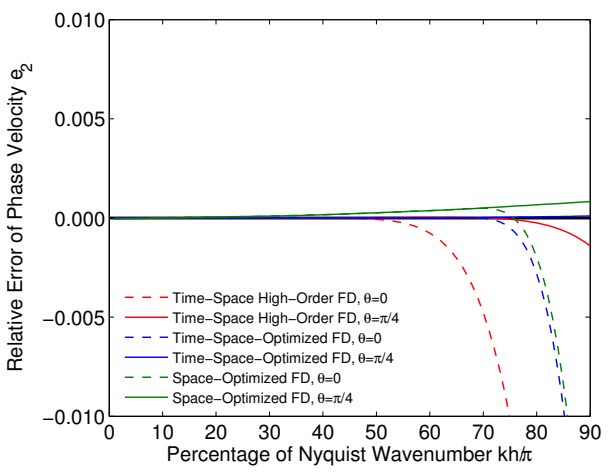

(a) $r=0.05$

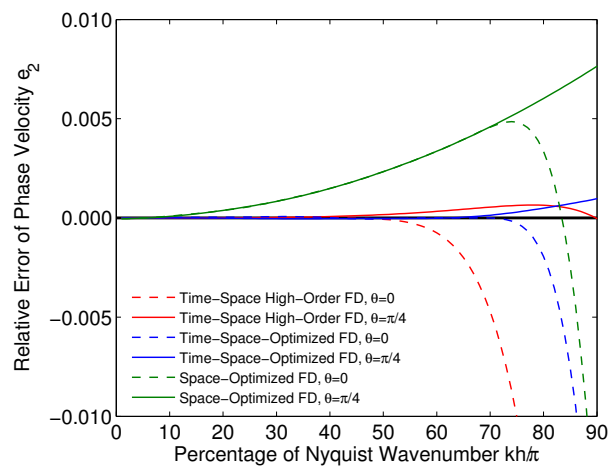

(b) $r=0.15$

Figure 4: Dispersion relations of the high-order scheme with coefficients given by eq. (5) (red curves), our optimized FD scheme (blue curves), and the space-domain optimized scheme obtained by minimizing eq. (17) (green curves) for 2D modeling with (a) $r=0.05$ and (b) $r=0.15$. Compared with the high-order FD scheme, our optimized FD scheme significantly extends the range of wavenumbers with accurate phase velocities. The scheme optimized in the space domain has similar dispersion curves to those of our optimized scheme in (a). However, a large time interval with $r=0.15$ generates significant time dispersion errors for the space-domain optimized scheme in (b), while our optimized scheme is able to suppress the errors for a wide range of wavenumbers. 


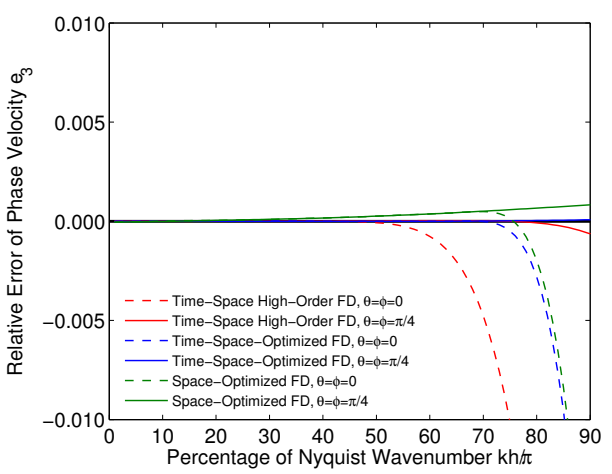

(a) $r=0.05$

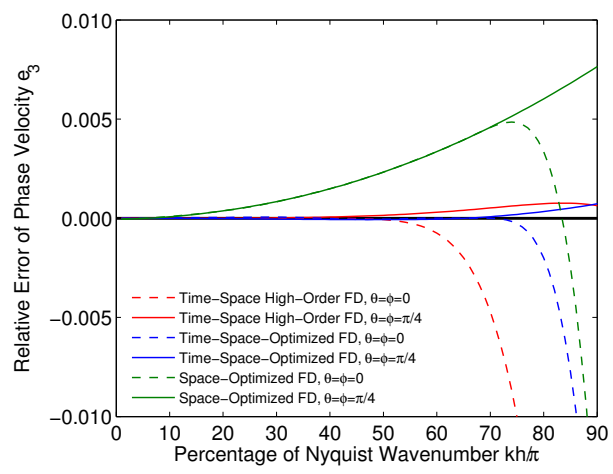

(b) $r=0.15$

Figure 5: Dispersion relations of the high-order scheme with coefficients given by eq. (7) (red curves), our optimized FD scheme (blue curves), and the space-domain optimized scheme obtained by minimizing eq. (17) (green curves) for 3D modeling with (a) $r=0.05$ and (b) $r=0.15$. The observations are similar to those made for the 2D case as shown in Fig. 4.

stencil use $3 M$ floating-point operations. When $2 M=16$, the computational cost for each time step for FD schemes using the new stencil is 1.21 and 1.38 times higher than that of FD schemes using the standard stencil for 2D and 3D modeling, respectively.

In summary, our optimized FD scheme is able to control dispersion errors for a much wider range of wavenumbers compared with its high-order counterpart. What makes our scheme distinct is that it generates accurate modeling results with a time interval approximately three times larger than that can be used in the optimized schemes using the standard stencil. Taking the computational cost for each time step into account, our optimized FD scheme speeds up the calculation by a factor of 2 to 2.5 compared with the optimized schemes using the standard stencil for a given $2 \mathrm{D}$ or $3 \mathrm{D}$ modeling problem.

\section{Numerical examples}

We conduct numerical modeling of 2D and 3D scalar-wave propagation in heterogeneous media, and compare the waveforms with reference ones. We use either 
analytical solutions or the high-order FD scheme with a fine time interval and a very high-order accuracy in space to compute the reference waveforms. In all the following examples, we compare the performance of three FD schemes including

- Scheme I : The optimized scheme in the space domain obtained by minimizing eq. (17) on the standard stencil;

- Scheme II : The high-order scheme with $16^{\text {th }}$-order accuracy in space and fourthorder accuracy in time; and

- Scheme III : Our optimized scheme in the time-space domain using the new stencil and objective functions (13) and (14).

According to the dispersion analysis, we expect Scheme III gives fewer time dispersion errors than Scheme I, and has better control of spatial dispersion errors than Scheme II.

Note that Scheme II is actually similar to a Lax-Wendroff scheme [e.g., 2, 20, 21]. The connections between the two schemes are discussed in detail in [19]. We have made extensive comparison between Scheme II and the standard high-order FD scheme in [19]. For media with a velocity contrast up to five, Scheme II is approximately two times faster than the standard high-order FD scheme to achieve the same modeling accuracy. Thus, we do not present the results obtained using the standard high-order FD scheme.

We keep the spatial grid spacing $h$ the same for Schemes I-III, and vary the time interval $\Delta t$ and the length of the FD operators $2 M$ to study the effect of dispersion errors. For the ease of comparison, we specify $\Delta t$ using the global CFL number $r_{\max }$ through $\Delta t=r_{\max } h / c_{\max }$ where $c_{\max }$ is the maximum wave speed in a model.

We use the coda-wave interferometry method to quantify the time shift between FD waveform $u_{1}(t)$ and reference waveform $u_{2}(t)$ [42]. We compute the time-shifted cross-correlation over a time window with a width of $t_{w}$ at the center time $t$ using

$$
R\left(t_{s}\right)=\frac{\int_{t-t_{w}}^{t+t_{w}} u_{1}\left(t^{\prime}\right) u_{2}\left(t^{\prime}+t_{s}\right) d t^{\prime}}{\left[\int_{t-t_{w}}^{t+t_{w}}\left(u_{1}\left(t^{\prime}\right)\right)^{2} d t^{\prime} \int_{t-t_{w}}^{t+t_{w}}\left(u_{2}\left(t^{\prime}+t_{s}\right)\right)^{2} d t^{\prime}\right]^{1 / 2}},
$$

where $t_{s}$ is the time shift. When $u_{2}(t)=u_{1}(t-\tau)$ is a time-shifted version of $u_{1}(t)$, $R\left(t_{s}\right)$ attains its maximum at $t_{s}=\tau$. In the general case, we obtain $t_{\max }$ as the time shift 
between two waveforms $u_{1}(t)$ and $u_{2}(t)$ when $R\left(t_{s}\right)$ attains its maximum at $t_{s}=t_{\max }$. When the two waveforms are identical, the maximum value of the time-shifted crosscorrelation is equal to unity at the zero lag time, i.e., $R\left(t_{s}=0\right)=1$.

The spectral content associated with seismic waves propagating in the Earth is generally modeled using a Ricker wavelet [43]

$$
s(t)=\left[1-2\left(\pi f_{0}\left(t-t_{0}\right)\right)^{2}\right] \exp \left[-\left(\pi f_{0}\left(t-t_{0}\right)\right)^{2}\right],
$$

where $f_{0}$ is the central frequency and $t_{0}=1 / f_{0}$. We use the Ricker wavelet as a source time function to generate scalar waves. Namely, we add the following source term to the right-hand side of the first equation in (2):

$$
\delta\left(\mathbf{x}-\mathbf{x}_{\mathbf{s}}\right) s(t),
$$

where $\mathbf{x}_{\mathbf{s}}$ is the location of the point source and $\delta(\cdot)$ is the Dirac delta function. A Ricker wavelet with $f_{0}=10 \mathrm{~Hz}$ is displayed in Fig. 6a. Figure 6b shows the broad spectrum content of the Ricker wavelet. The maximum frequency is approximately $2.5 f_{0}$.

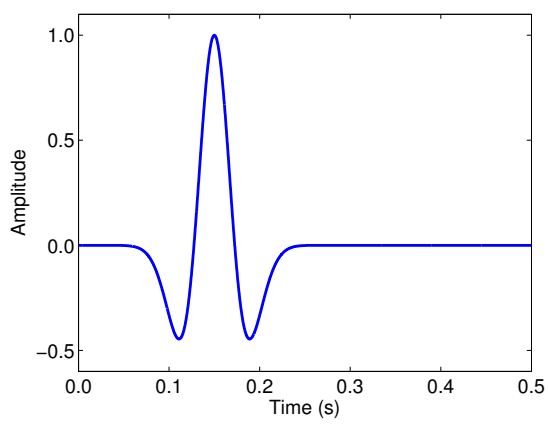

(a) Time domain

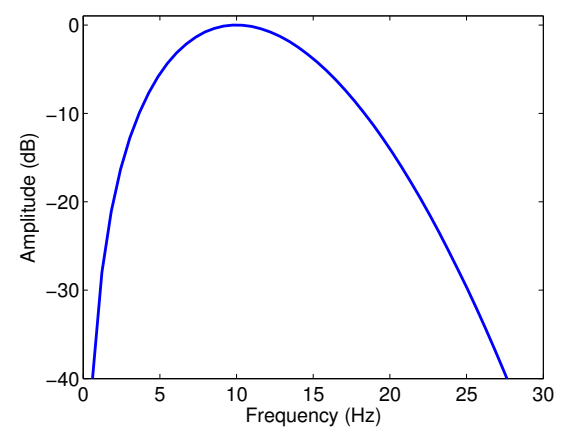

(b) Frequency domain

Figure 6: The Ricker wavelet shown in (a) time and (b) frequency domains. The central frequency is $f_{0}=10 \mathrm{~Hz}$. 


\section{1. $2 D$ examples}

\subsubsection{Two-layer model}

Our first example is modeling of scalar-wave propagation in a 2D layered velocity model defined by

$$
c(x, z)= \begin{cases}3000 \mathrm{~m} / \mathrm{s}, & 0 \mathrm{~m} \leq z \leq 3000 \mathrm{~m} ; \\ 3300 \mathrm{~m} / \mathrm{s}, & 3000 \mathrm{~m}<z \leq 4000 \mathrm{~m} .\end{cases}
$$

The model is defined on a 2D grid with $x \in[-2000 \mathrm{~m}, 2000 \mathrm{~m}]$ and $z \in[0 \mathrm{~m}, 4000 \mathrm{~m}]$. The spatial grid spacing of the model is $h=10 \mathrm{~m}$. A Ricker-wavelet source with a central frequency of $45 \mathrm{~Hz}$ is located at $(-1534 \mathrm{~m}, 50 \mathrm{~m})$. The receiver is located at $(0 \mathrm{~m}$, $1850 \mathrm{~m}$ ). Figures $7 \mathrm{a}$ and $7 \mathrm{~b}$ display the reflected waveforms calculated using Scheme I with $2 M=16$, together with the analytical waveform. The spatial dispersion errors are very small, because of the optimization. However, using the large time interval with $r_{\max }=0.15$ gives huge time dispersion errors as depicted in Fig. 7a. A small time interval with $r_{\max }=0.05$ has to be used to suppress the errors as displayed in Fig. 7b. Scheme II with $2 M=16$ is capable of suppressing temporal dispersion errors using a large time interval with $r_{\max }=0.15$, as shown in Fig. 7c, but the wiggles following the reflected waveform indicate that the waveform contains significant spatial dispersion errors. The wiggles disappear in Fig. 7d when we increase the length of the FD operator to $2 M=32$ for Scheme II. Scheme III with $2 M=16$ is able to accurately generate the waveform using a large time interval with $r_{\max }=0.15$, as displayed in Fig. 7e.

The errors between the simulated waveforms and the analytical waveforms are quantified in Table 1 . The relative time shift $t_{\max } / T_{0}$, where $T_{0}$ is the central period of waveforms, and the time-shifted cross-correlation $R\left(t_{\max }\right)$ obtained using eq. (18) mainly measure time dispersion errors. The relative time-shift error is $8.6 \%$ for Scheme I with $r_{\max }=0.15$ and the resulting time-shifted cross-correlation is 0.946 . The relative time-shift error is below 1.0\% and the time-shifted cross-correlation is at least 0.994 for all the other schemes. To measure the energy of the spurious oscillations caused by spatial dispersion errors, we define the $L^{2}$-error $\varepsilon_{2}$ using

$$
\varepsilon_{2}=\sqrt{\int_{1.43}^{1.5}\left(u(t)-u_{\text {anal }}(t)\right)^{2} d t},
$$




\begin{tabular}{|l|c|c|c|c|}
\hline & $t_{\max } / T_{0}$ & $R\left(t_{\max }\right)$ & $\varepsilon_{2}$ & Cost \\
\hline Scheme I with $r_{\max }=0.15$ and $2 M=16$ & $8.6 \%$ & 0.946 & $4.58 \mathrm{E}-4$ & 0.8 \\
\hline Scheme I with $r_{\max }=0.05$ and $2 M=16$ & $0.9 \%$ & 0.997 & $7.77 \mathrm{E}-4$ & 2.5 \\
\hline Scheme II with $r_{\max }=0.15$ and $2 M=16$ & $0.0 \%$ & 0.994 & $4.02 \mathrm{E}-3$ & 1.0 \\
\hline Scheme II with $r_{\max }=0.15$ and $2 M=32$ & $0.9 \%$ & 0.997 & $7.77 \mathrm{E}-4$ & 2.0 \\
\hline Scheme III with $r_{\max }=0.15$ and $2 M=16$ & $0.0 \%$ & 0.997 & $5.62 \mathrm{E}-4$ & 1.0 \\
\hline
\end{tabular}

Table 1: Quantified errors between the simulated waveforms and the analytical waveforms depicted in Fig. 7. The time shift $t_{\max }$ and the time-shifted cross-correlation $R\left(t_{\max }\right)$ are obtained using eq. (18). $T_{0}=1 / f_{0}$ is the central wave period. The $L^{2}$-error $\varepsilon_{2}$ is defined in eq. (20). The last column lists the estimated computational cost of all FD schemes relative to Scheme III. The computational cost of Scheme III is only $40 \%$ of that of Scheme I with $r_{\max }=0.05$ and $2 M=16$, and $50 \%$ of that of Scheme II with $r_{\max }=0.15$ and $2 M=32$. Moreover, Scheme III generates the most accurate waveform.

where $u(t)$ is the calculated waveform using FD schemes and $u_{\text {anal }}(t)$ is the analytical waveform. The $L^{2}$-error $\varepsilon_{2}$ is $4.02 \mathrm{E}-3$ for Scheme II with $r_{\max }=0.15$ and $2 M=16$, while the error is at most 7.77E-4 for all the other schemes. The last column of Table 1 lists the estimated computational cost of all FD schemes relative to Scheme III. The computational cost of Scheme III is only $40 \%$ of that of Scheme I with $r_{\max }=0.05$ and $2 M=16$, and $50 \%$ of that of Scheme II with $r_{\max }=0.15$ and $2 M=32$. Moreover, Scheme III generates the most accurate waveform.

\subsubsection{D SEG/EAGE salt model}

Next, we compare the accuracy and efficiency of the three FD schemes for a 2D SEG/EAGE salt model depicted in Fig. 8. The model is defined on a 2D grid with $x \in[-7500 \mathrm{~m}, 7500 \mathrm{~m}]$ and $z \in[0 \mathrm{~m}, 3975 \mathrm{~m}]$. The spatial grid spacing of the model is $h=25 \mathrm{~m}$. A Ricker-wavelet source with a central frequency of $10 \mathrm{~Hz}$ is located at ($6000 \mathrm{~m}, 125 \mathrm{~m}$ ), and the receiver array is located at the same depth. We use Scheme II with a fine time interval with $r_{\max }=0.05$ and a very high-order accuracy in space 


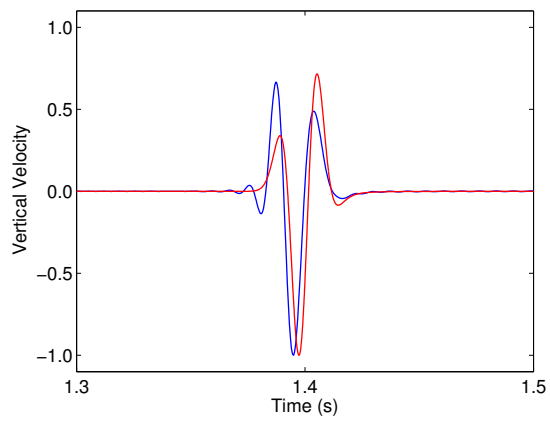

(a) Scheme I with $r_{\max }=0.15$ and $2 M=16$

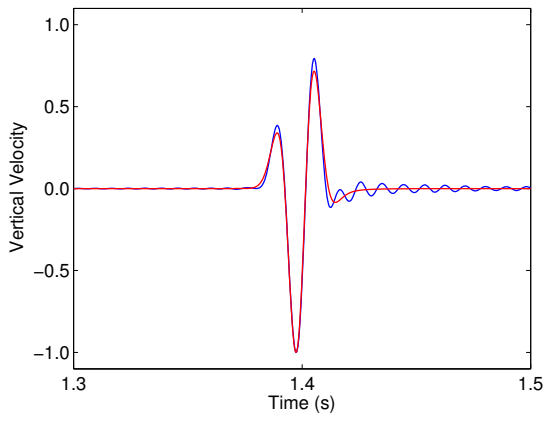

(c) Scheme II with $r_{\max }=0.15$ and $2 M=16$

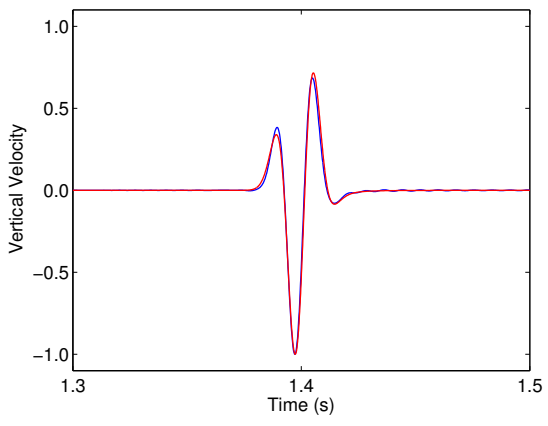

(b) Scheme I with $r_{\max }=0.05$ and $2 M=16$

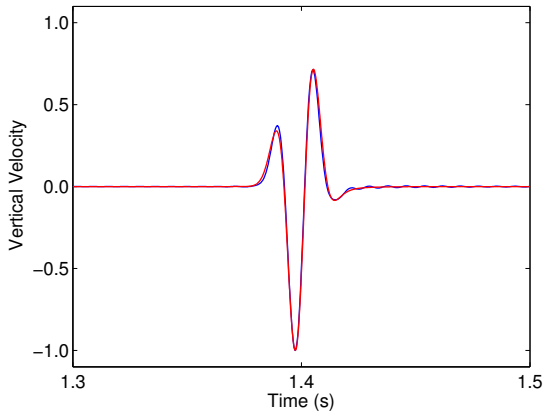

(d) Scheme II with $r_{\max }=0.15$ and $2 M=32$

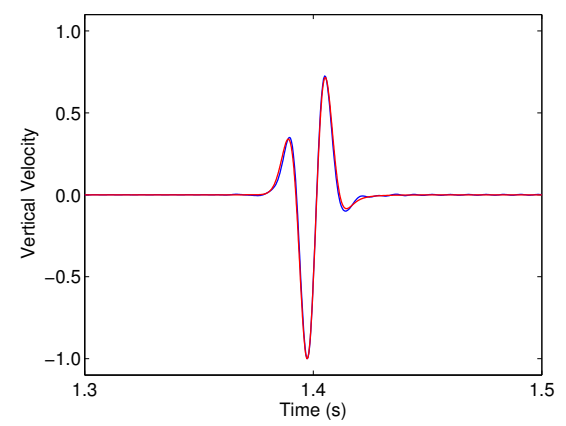

(e) Scheme III with $r_{\max }=0.15$ and $2 M=16$

Figure 7: Reflected waveforms calculated using Scheme I (first row), Scheme II (second row), and Scheme III (third row) for the 2D layered model (19). The red line is the analytical waveform; the blue line is the simulated waveform. (a) and (c) contain significant numerical dispersion. Satisfactory modeling results are displayed in (b), (d), and (e), among which the computational cost of (e) is the lowest. 
$(2 M=48)$ to generate reference waveforms. Figure 9a demonstrates the common-shot gather calculated using Scheme II with $r_{\max }=0.5$ and $2 M=16$. The four-timeamplified error between the FD common-shot gather and the reference one is shown in Fig. 9c. We observe wiggles caused by spatial dispersion errors. Scheme III with the same values of $r_{\max }$ and $M$ successfully suppresses the errors, as shown in Figs. $9 \mathrm{~b}$ and 9 d.

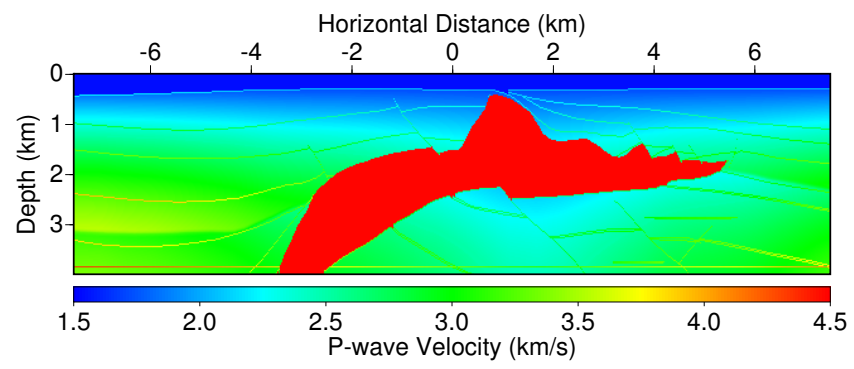

Figure 8: 2D SEG/EAGE salt model for scalar-wave modeling.

Figure 10 displays the waveforms calculated using the three schemes together with the reference waveform at a receiver with an offset (source-receiver distance) of $1375 \mathrm{~m}$ or approximately 20 minimum wavelengths. The dispersion errors are quantified in Fig. 11. The time shift between the calculated waveform and the reference waveform in Fig. 10a is due to time dispersion errors of Scheme I with $r_{\max }=0.4$ and $2 M=$ 16. We have to reduce the time interval by half to suppress the errors, as shown in Fig. 10b. Scheme II is able to handle a large time interval with $r_{\max }=0.5$, but it suffers from significant spacial dispersion errors if we use $2 M=16$ (Fig. 10c). The spacial dispersion errors are mostly reduced if we increase the length of the FD operator to $2 M=32$ (Fig. 10d). Scheme III with $r_{\max }=0.5$ and $2 M=16$ gives the most accurate waveform (Fig. 10e) among all the schemes. The computational cost of Scheme III is approximately a half of that of Scheme I with $r_{\max }=0.2$ and $2 M=16$ or Scheme II with $r_{\max }=0.5$ and $2 M=32$.

The advantage of Scheme III is more obvious if we compare the simulated waveforms and the reference waveform at a receiver with a larger offset of $9750 \mathrm{~m}$ or approximately 160 minimum wavelengths in Fig. 12. The dispersion errors are quanti- 


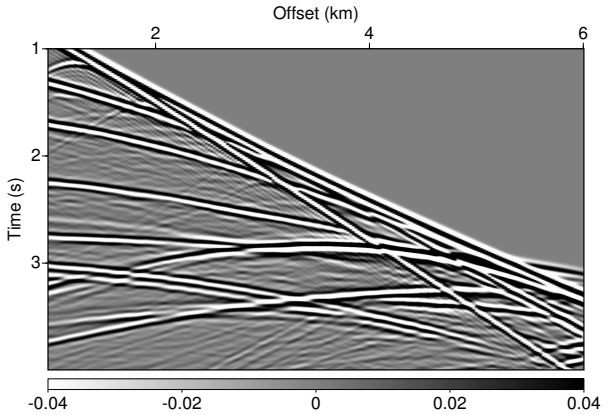

(a) Scheme II with $r_{\max }=0.5$ and $2 M=16$

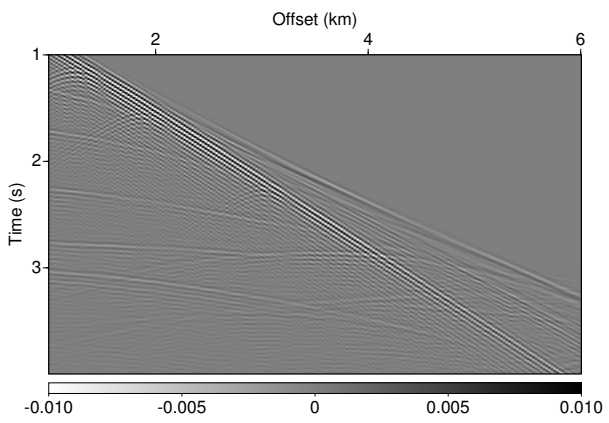

(c) Four-time-amplified error of (a)

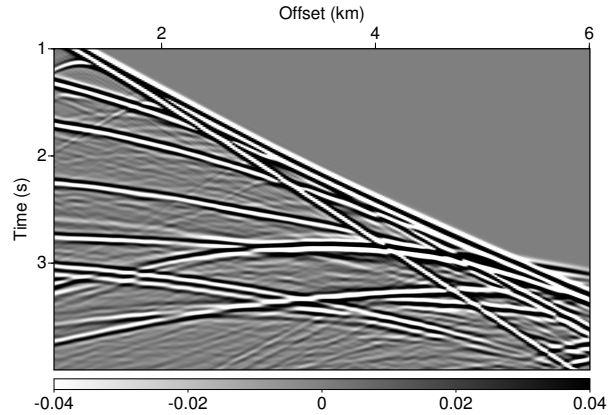

(b) Scheme III with $r_{\max }=0.5$ and $2 M=16$

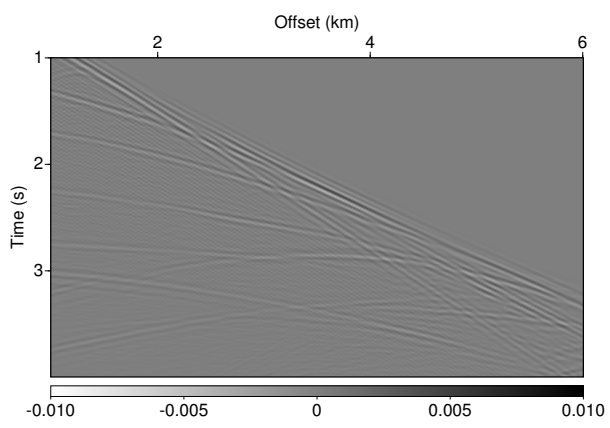

(d) Four-time-amplified error of (b)

Figure 9: Common-shot gathers calculated using (a) Scheme II and (b) Scheme III with $r_{\max }=0.5$ and $2 M=16$ for the 2D SEG/EAGE salt model. (c) and (d) are the four-time-amplified errors of (a) and (b) compared with the reference. Scheme II leads to significant spatial dispersion errors as shown in (c), while Scheme III suppresses the errors as shown in (d). 


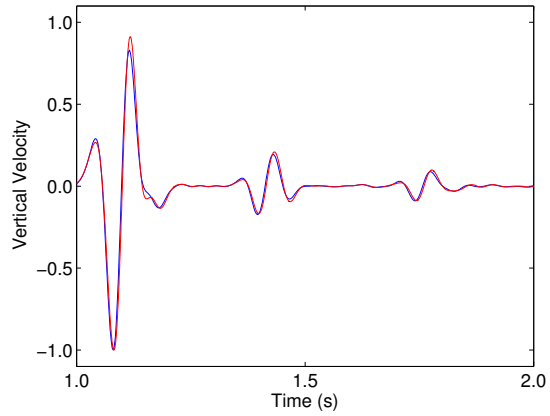

(a) Scheme I with $r_{\max }=0.4$ and $2 M=16$

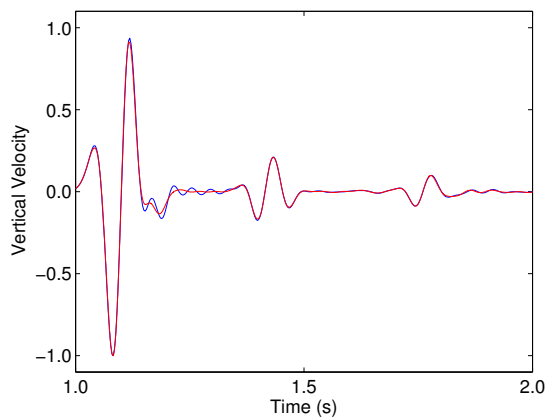

(c) Scheme II with $r_{\max }=0.5$ and $2 M=16$

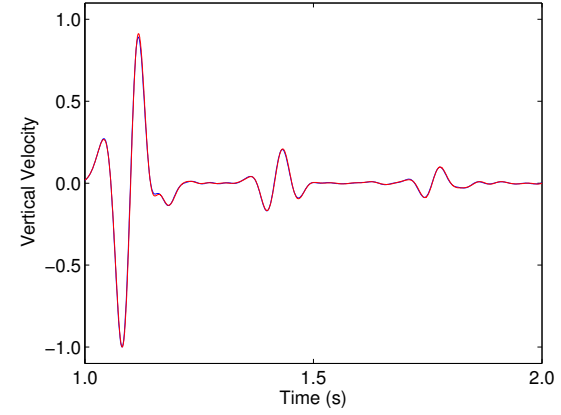

(b) Scheme I with $r_{\max }=0.2$ and $2 M=16$

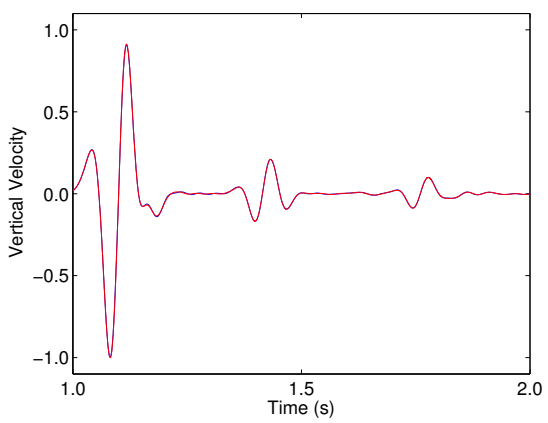

(d) Scheme II with $r_{\max }=0.5$ and $2 M=32$

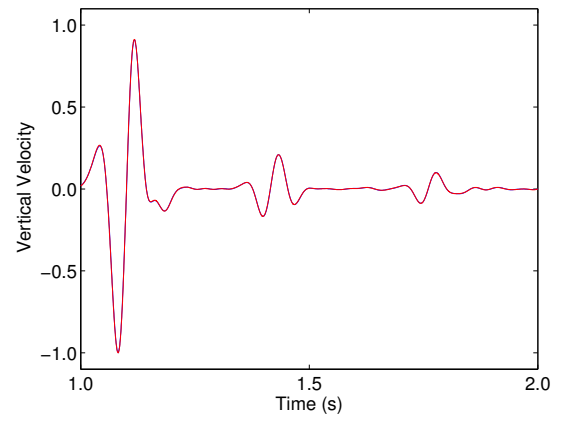

(e) Scheme III with $r_{\max }=0.5$ and $2 M=16$

Figure 10: Waveforms (blue lines) at a receiver with an offset of $1375 \mathrm{~m}$ or approximately 20 minimum wavelengths calculated using Scheme I (first row), Scheme II (second row), and Scheme III (third row) for the 2D SEG/EAGE salt model. The red line is the reference waveform. Both (a) and (c) suffer from significant dispersion errors. We have to decrease the time interval for Scheme I in (b) or increase the length of the FD operator for Scheme II in (d) to suppress the errors. Scheme III gives a well-matched waveform in (e) with approximately a half of the computational cost compared with Scheme I in (b) or Scheme2t in (d). 


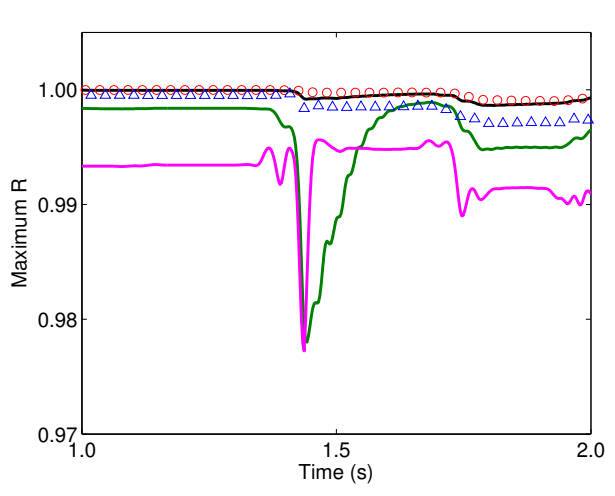

(a) $R\left(t_{\max }\right)$

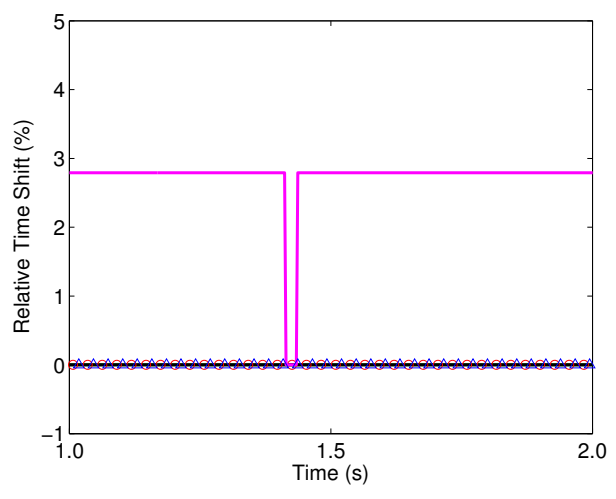

(b) $t_{\max } / T_{0}$

Figure 11: (a) Time-shifted cross-correlation $R\left(t_{\max }\right)$ and (b) relative time shift $t_{\max } / T_{0}$ between the calculated waveform and the reference waveform at a receiver with an offset of $1375 \mathrm{~m}$ or approximately 20 minimum wavelengths for the 2D SEG/EAGE salt model. Magenta line: Scheme I with $r_{\max }=0.4$ and $2 M=16$; Blue symbols: Scheme I with $r_{\max }=0.2$ and $2 M=16$; Dark green line: Scheme II with $r_{\max }=0.5$ and $2 M=16$; Black line: Scheme II with $r_{\max }=0.5$ and $2 M=32$; Red symbols: Scheme III with $r_{\max }=0.5$ and $2 M=16$. The width of the time window is $t_{w}=2 T_{0}$ in eq. (18), where $T_{0}$ is the central wave period. Scheme III generates the most accurate waveform among the three schemes. 
fied in Fig. 13. The errors of Scheme I and II become larger when the waves propagate longer distance. In particular, a huge time shift occurs in the waveform calculated using Scheme I with $r_{\max }=0.4$ and $2 M=16$ (Fig. 12a). By contrast, Scheme III maintains its excellent performance (Fig. 12e).

\section{2. $3 D$ examples}

\subsubsection{Two-layer model}

We simulate scalar-wave propagation in 3D models, and compare the accuracy and efficiency of the three schemes. We consider a layered model defined by

$$
c(x, y, z)= \begin{cases}3000 \mathrm{~m} / \mathrm{s}, & 0 \mathrm{~m} \leq z \leq 2600 \mathrm{~m} ; \\ 3300 \mathrm{~m} / \mathrm{s}, & 2600 \mathrm{~m}<z \leq 3000 \mathrm{~m} .\end{cases}
$$

The model is defined on a 3D grid with $x \in[-2000 \mathrm{~m}, 2000 \mathrm{~m}], y \in[-2000 \mathrm{~m}, 2000 \mathrm{~m}]$, and $z \in[0 \mathrm{~m}, 3000 \mathrm{~m}]$. The spatial grid spacing of the model is $h=10 \mathrm{~m}$. A Rickerwavelet source with a central frequency of $48 \mathrm{~Hz}$ is located at $(-1000 \mathrm{~m}, 1000 \mathrm{~m}, 50 \mathrm{~m})$. The receiver is located at $(0 \mathrm{~m}, 0 \mathrm{~m}, 1400 \mathrm{~m})$. Figure 14 displays the reflected waveform calculated using the three schemes, together with the analytical waveform. The errors are quantified in Table 2 . The $L^{2}$-error $\varepsilon_{3}$ is defined by

$$
\varepsilon_{3}=\sqrt{\int_{1.27}^{1.35}\left(u(t)-u_{\text {anal }}(t)\right)^{2} d t},
$$

where $u(t)$ is the calculated waveform using FD schemes and $u_{\text {anal }}(t)$ is the analytical waveform. The last column of the table lists the estimated computational cost of all FD schemes relative to Scheme III. When $r=0.15$ and $2 M=16$, Scheme I and II give significant dispersion errors (Figs. 14a and 14c), while Scheme III generates the waveform with very small errors (Fig. 14e). Accurate waveforms can also be obtained by reducing the time interval for Scheme I or by increasing the length of the FD operator for Scheme II, as shown in Figs. 14b and 14d. Scheme III is the best choice among the three schemes because it speeds up the 3D modeling by a factor of approximately two relative to the others. 


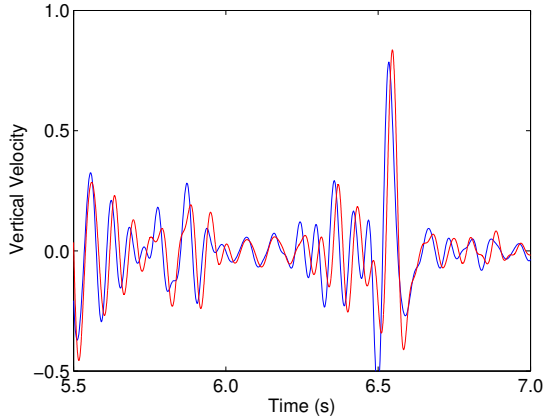

(a) Scheme I with $r_{\max }=0.4$ and $2 M=16$

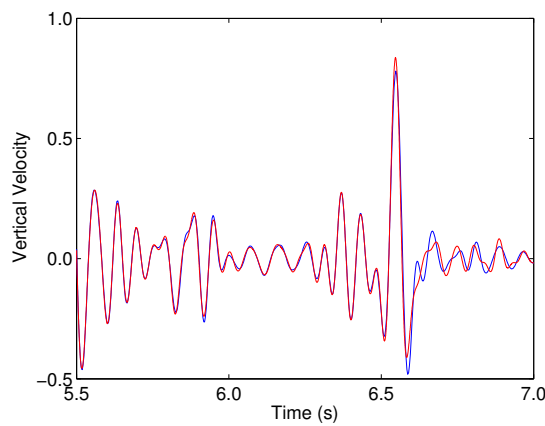

(c) Scheme II with $r_{\max }=0.5$ and $2 M=16$

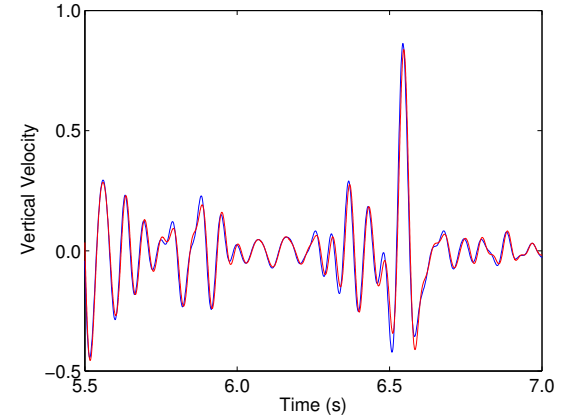

(b) Scheme I with $r_{\max }=0.2$ and $2 M=16$

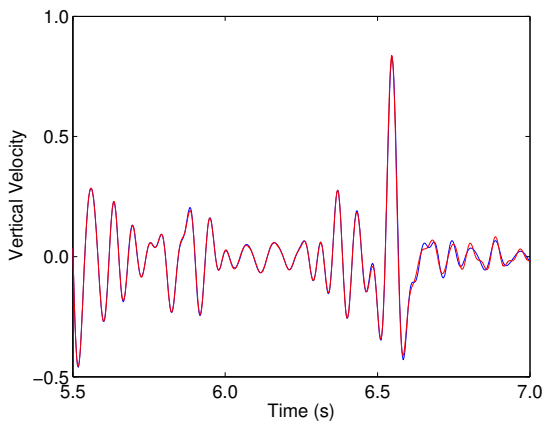

(d) Scheme II with $r_{\max }=0.5$ and $2 M=32$

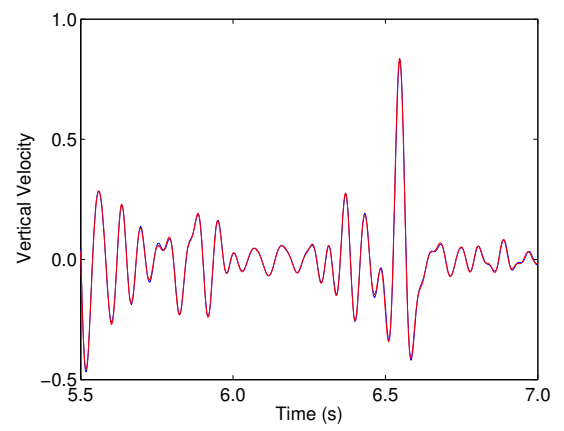

(e) Scheme III with $r_{\max }=0.5$ and $2 M=16$

Figure 12: Waveforms (blue lines) at a receiver with an offset of $9750 \mathrm{~m}$ or approximately 160 minimum wavelengths calculated using Scheme I (first row), Scheme II (second row), and Scheme III (third row) for the 2D SEG/EAGE salt model. The red line is the reference waveform. Compared with Fig. 10, the errors of Scheme I and II become larger when the waves propagate longer distance, while Scheme III maintains its excellent performance. 


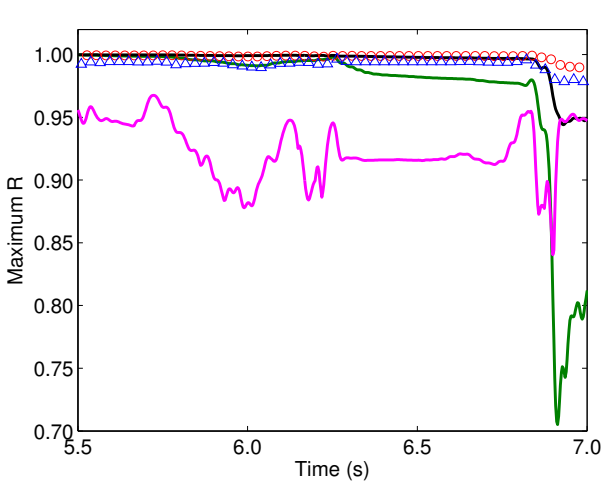

(a) $R\left(t_{\max }\right)$

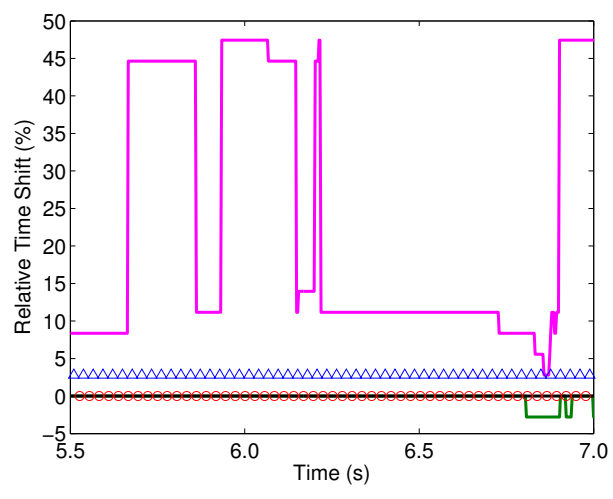

(b) $t_{\max } / T_{0}$

Figure 13: (a) Time-shifted cross-correlation $R\left(t_{\max }\right)$ and (b) relative time shift $t_{\max } / T_{0}$ between the calculated waveform and the reference waveform at a receiver with an offset of $9750 \mathrm{~m}$ or approximately 160 minimum wavelengths for the 2D SEG/EAGE salt model. Magenta line: Scheme I with $r_{\max }=0.4$ and $2 M=16$; Blue symbols: Scheme I with $r_{\max }=0.2$ and $2 M=16$; Dark green line: Scheme II with $r_{\max }=0.5$ and $2 M=16$; Black line: Scheme II with $r_{\max }=0.5$ and $2 M=32$; Red symbols: Scheme III with $r_{\max }=0.5$ and $2 M=16$. The width of the time window is $t_{w}=2 T_{0}$ in eq. (18), where $T_{0}$ is the central wave period. Scheme III gives the most accurate waveform among the three schemes and is the most efficient. 


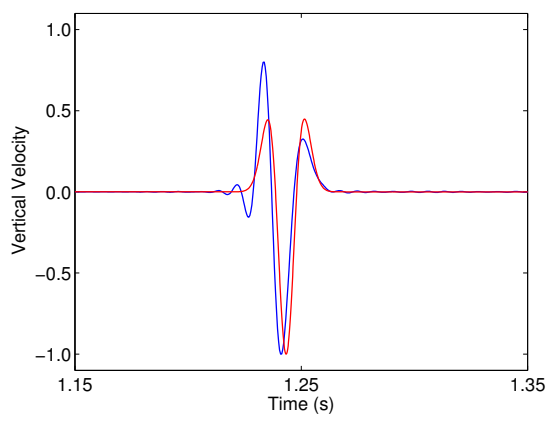

(a) Scheme I with $r_{\max }=0.15$ and $2 M=16$

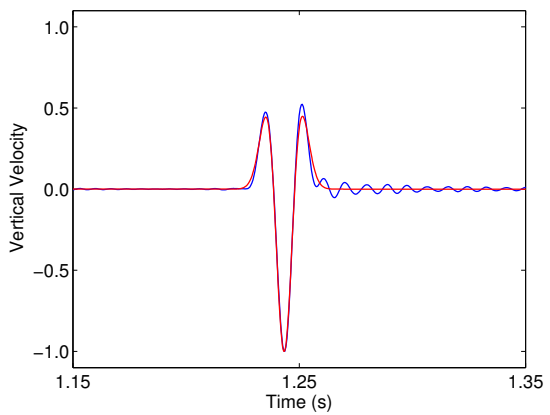

(c) Scheme II with $r_{\max }=0.15$ and $2 M=16$

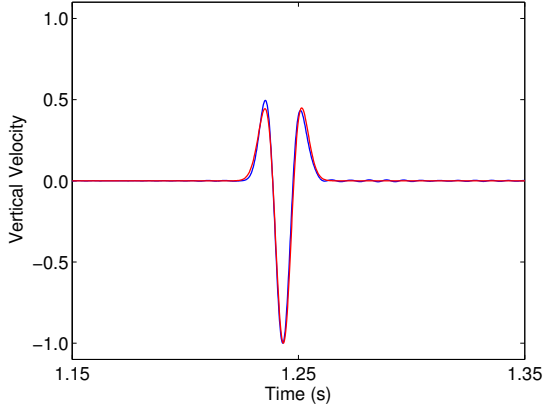

(b) Scheme I with $r_{\max }=0.05$ and $2 M=16$

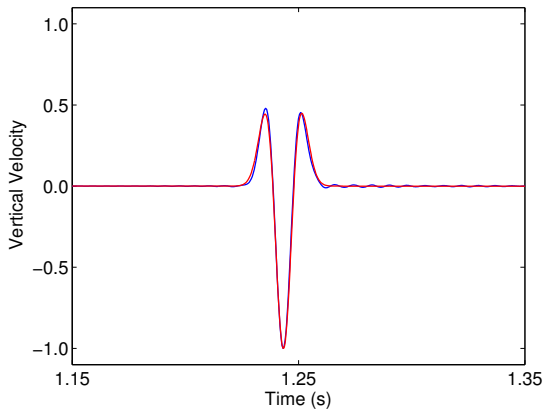

(d) Scheme II with $r_{\max }=0.15$ and $2 M=32$

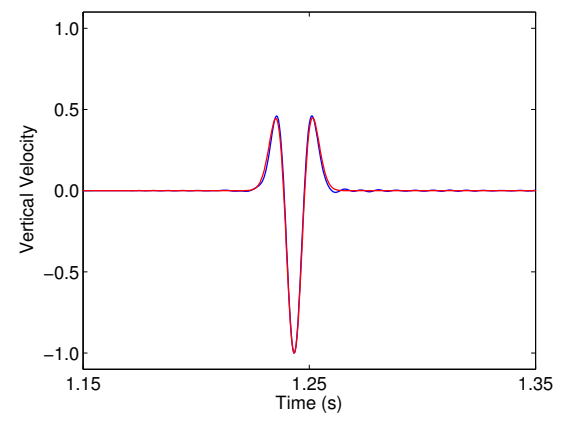

(e) Scheme III with $r_{\max }=0.15$ and $2 M=16$

Figure 14: Reflected waveform calculated using Scheme I (first row), Scheme II (second row), and Scheme III (third row) for the 3D layered model (21). The red line is the analytical waveform; the blue line is the simulated waveform. (a) and (c) contain significant numerical dispersion. The waveforms in (b), (d) and (e) have similar quality, but the computational cost of (e) is the lowest. 


\begin{tabular}{|l|c|c|c|c|}
\hline & $t_{\max } / T_{0}$ & $R\left(t_{\max }\right)$ & $\varepsilon_{2}$ & Cost \\
\hline Scheme I with $r_{\max }=0.15$ and $2 M=16$ & $8.2 \%$ & 0.953 & $6.50 \mathrm{E}-4$ & 0.7 \\
\hline Scheme I with $r_{\max }=0.05$ and $2 M=16$ & $1.0 \%$ & 0.997 & $7.80 \mathrm{E}-4$ & 2.2 \\
\hline Scheme II with $r_{\max }=0.15$ and $2 M=16$ & $0.0 \%$ & 0.993 & $4.12 \mathrm{E}-3$ & 1.0 \\
\hline Scheme II with $r_{\max }=0.15$ and $2 M=32$ & $0.5 \%$ & 0.997 & $9.75 \mathrm{E}-4$ & 2.0 \\
\hline Scheme III with $r_{\max }=0.15$ and $2 M=16$ & $0.0 \%$ & 0.997 & $6.67 \mathrm{E}-4$ & 1.0 \\
\hline
\end{tabular}

Table 2: Quantified errors between the simulated waveform and the analytical waveform depicted in Fig. 14. $T_{0}=1 / f_{0}$ is the central wave period. The $L^{2}$-error $\varepsilon_{3}$ is defined in eq. (22). The last column lists the estimated computational cost of all FD schemes relative to Scheme III. To achieve the same modeling accuracy, Scheme III is the most efficient among all the schemes.

\subsubsection{D SEG/EAGE salt model}

Our last modeling example is simulation of scalar-wave propagation in a 3D SEG/EAGE salt model shown in Fig. 15. The model is defined on a 3D grid with $x \in[-6500 \mathrm{~m}, 6500 \mathrm{~m}]$, $y \in[-6500 \mathrm{~m}, 6500 \mathrm{~m}]$, and $z \in[0 \mathrm{~m}, 4180 \mathrm{~m}]$. The spatial grid spacing of the model is $h=20 \mathrm{~m}$. A Ricker-wavelet source with a central frequency of $13 \mathrm{~Hz}$ is located at (-5000 m, -5000 m, $100 \mathrm{~m})$. Reference waveforms are generated using Scheme II with a fine time interval with $r_{\max }=0.05$ and a very high-order accuracy in space $(2 M=48)$. The common-shot gather calculated using Scheme II with $r_{\max }=0.38$ and $2 M=16$ contains spurious oscillations caused by spatial dispersion errors, as shown in Figs.16a and 16c. Scheme III with the same values of $r_{\max }$ and $M$ mostly removes the oscillations, as displayed in Figs. 16b and 16d.

In Fig. 17, we compare the waveforms calculated using the three schemes at a receiver with an offset of $7500 \mathrm{~m}$ in $x$-direction with the reference waveform. Scheme I with $r_{\max }=0.38$ gives severe time shift between the simulated waveform and the reference waveform, as shown in Fig. 17a. A small time interval with $r_{\max }=0.15$ is needed for Scheme I to eliminate the time shift (Fig. 17b). Scheme II with $r_{\max }=0.38$ and $2 M=16$ suffers from spatial dispersion errors, but not time dispersion errors (Fig. 17c). The spatial dispersion errors can be greatly reduced by increasing the length 
of the FD stencil to $2 M=32$ (Fig. 17d). The waveform generated using Scheme III with $r_{\max }=0.38$ and $2 M=16$ contains very small numerical dispersion (Fig. 17e). According to the quantified errors shown in Fig. 18, Scheme III is able to achieve similar modeling accuracy with only approximately a half of the computational cost compared with the other two schemes.

\section{Conclusions}

We have developed a novel finite-difference scheme optimized in the time-space domain for modeling 2D and 3D scalar-wave propagation in geophysical problems. Our optimized finite-difference scheme uses a new finite-difference stencil containing a few more grid points than the standard stencil. We have obtained optimized finitedifference coefficients by minimizing an objective function of relative errors of the phase velocity. Our new optimized scheme is capable of suppressing dispersion errors for a wide range of wavenumbers using a large time interval. In addition, the new scheme is accurate for waves propagating along all directions. We have validated the improved accuracy and computational efficiency of our optimized finite-difference scheme using both dispersion analysis and numerical modeling of scalar-wave propagation in $2 \mathrm{D}$ and $3 \mathrm{D}$ complex media. To achieve the same modeling accuracy, our

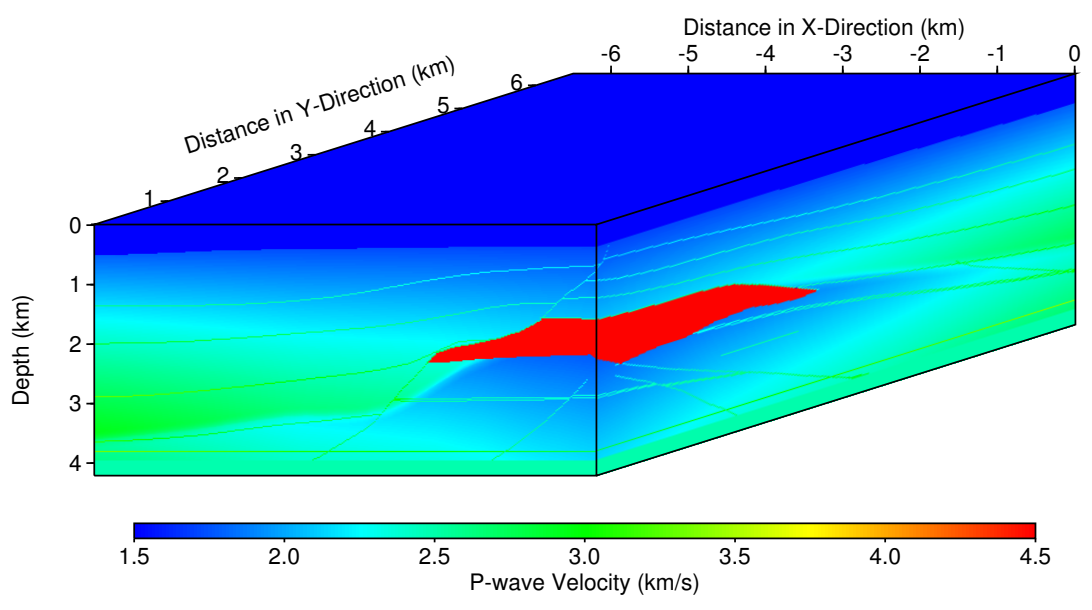

Figure 15: 3D SEG/EAGE salt model for scalar-wave modeling. 


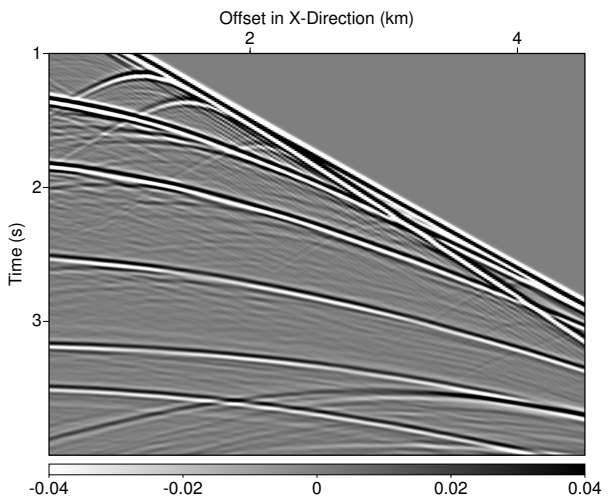

(a) Scheme II with $r_{\max }=0.38$ and $2 M=16$

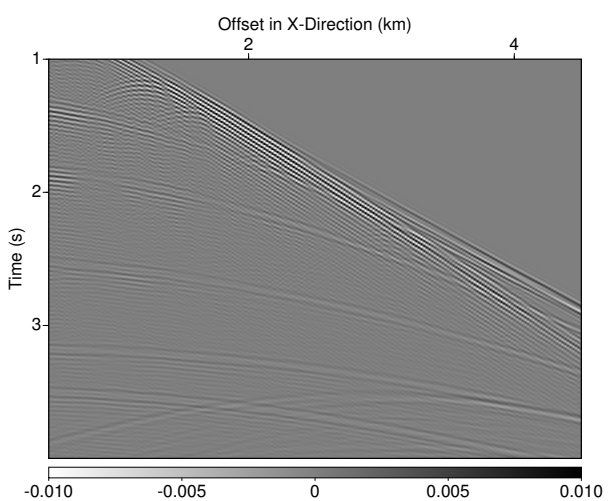

(c) Four-time-amplified error of (a)

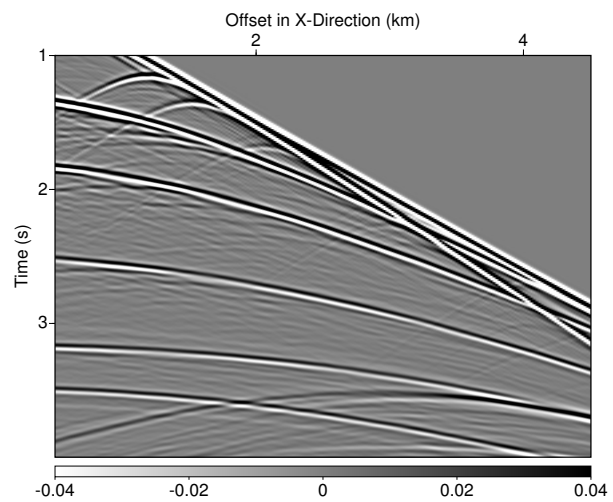

(b) Scheme III with $r_{\max }=0.38$ and $2 M=16$

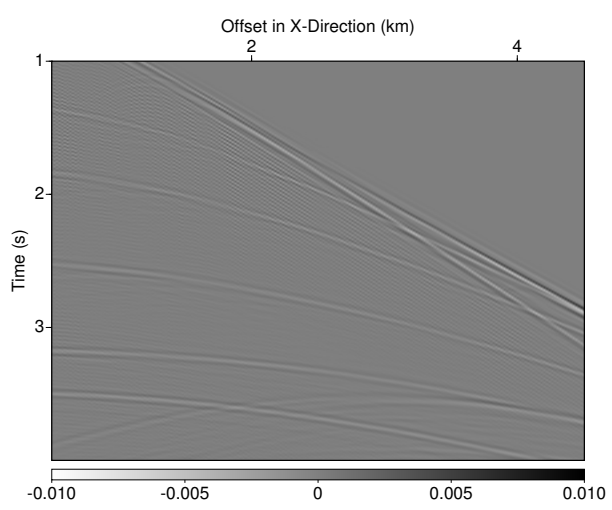

(d) Four-time-amplified error of (b)

Figure 16: Common-shot gathers calculated using (a) Scheme II and (b) Scheme III with $r_{\max }=0.38$ and $2 M=16$ for the 3D SEG/EAGE salt model. (c) and (d) are the four-time-amplified errors of (a) and (b) compared with the reference. The receiver array is along the $x$-axis. Scheme II generates significant spatial dispersion errors as shown in (c), while Scheme III successfully suppresses the errors as shown in (d). 


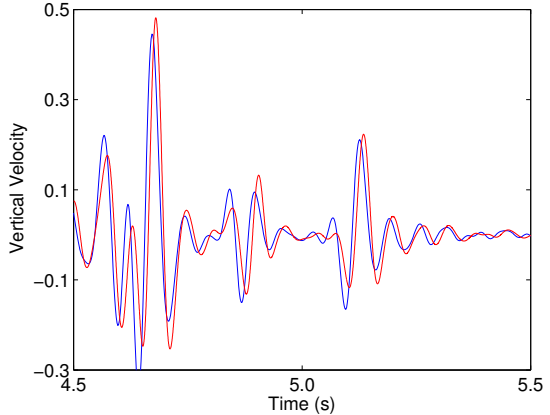

(a) Scheme I with $r_{\max }=0.38$ and $2 M=16$

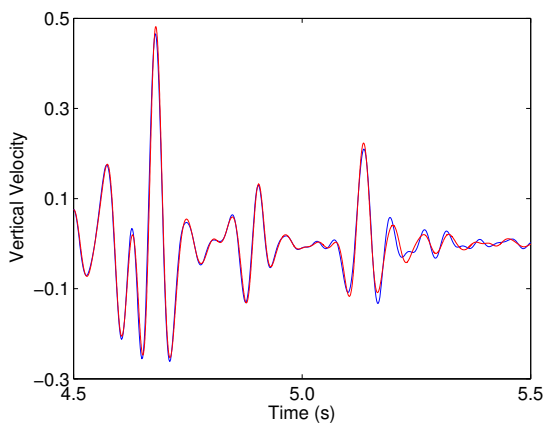

(c) Scheme II with $r_{\max }=0.38$ and $2 M=16$

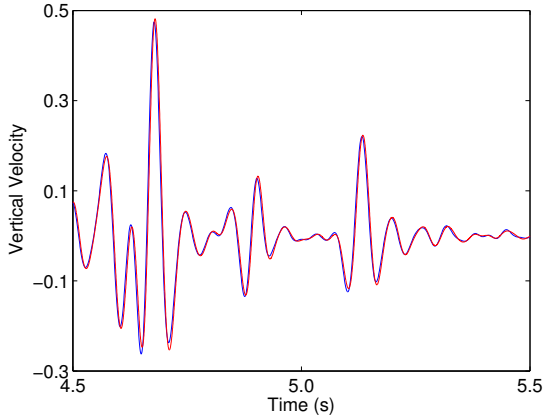

(b) Scheme I with $r_{\max }=0.15$ and $2 M=16$

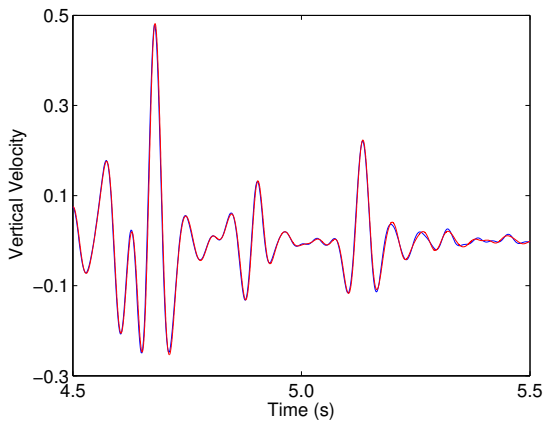

(d) Scheme II with $r_{\max }=0.38$ and $2 M=32$

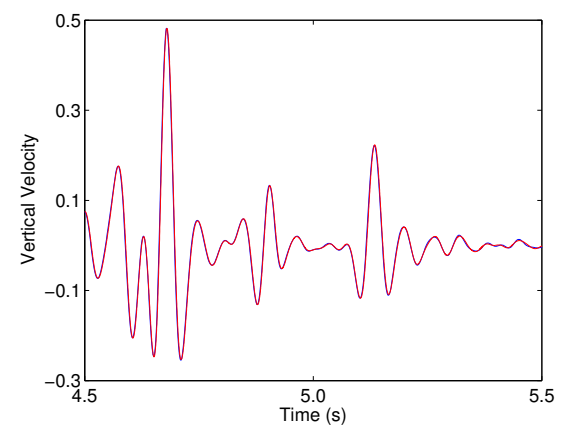

(e) Scheme III with $r_{\max }=0.38$ and $2 M=16$

Figure 17: Waveforms (blue lines) at a receiver with an offset of $7500 \mathrm{~m}$ in $x$-direction calculated using Scheme I (first row), Scheme II (second row), and Scheme III (third row) for the 3D SEG/EAGE salt model. The red line is the reference waveform. Both (a) and (c) suffer from dispersion errors. We have to decrease the time interval for Scheme I in (b) or increase the length of the FD stencil for Scheme II in (d) to suppress the errors. Scheme III gives a well-matched waveform in (e) with only approximately a half of the computational cost compared with Scheme I or II. 


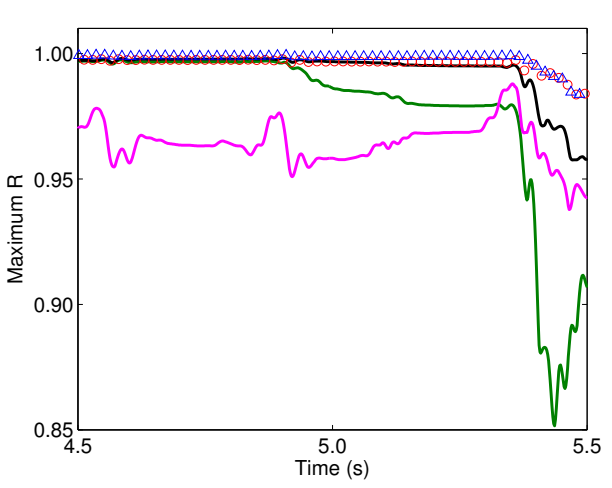

(a) $R\left(t_{\max }\right)$

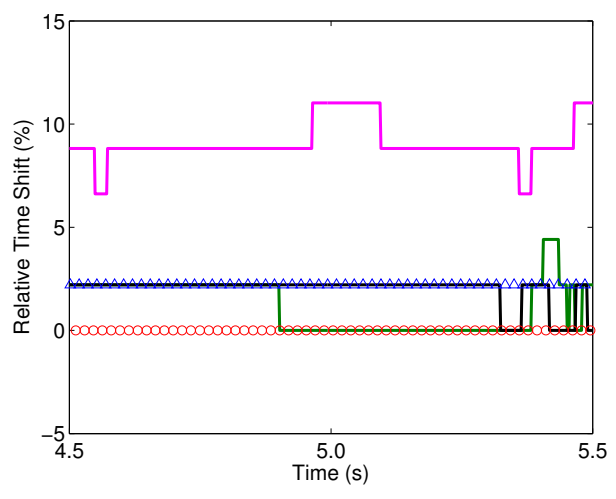

(b) $t_{\max } / T_{0}$

Figure 18: (a) Time-shifted cross-correlation $R\left(t_{\max }\right)$ and (b) relative time shift $t_{\max } / T_{0}$ between the calculated waveform and the reference waveform at a receiver with an offset of $7500 \mathrm{~m}$ in $x$-direction for the 3D SEG/EAGE salt model. Magenta line: Scheme I with $r_{\max }=0.38$ and $2 M=16$; Blue symbols: Scheme I with $r_{\max }=0.15$ and $2 M=16$; Dark green line: Scheme II with $r_{\max }=0.38$ and $2 M=16$; Black line: Scheme II with $r_{\max }=0.38$ and $2 M=32$; Red symbols: Scheme III with $r_{\max }=0.38$ and $2 M=16$. The width of the time window is $t_{w}=2 T_{0}$ in eq. (18), where $T_{0}$ is the central wave period. Scheme III is able to achieve similar modeling accuracy with only approximately a half of the computational cost compared with the other two schemes. 
optimized scheme is computationally two times faster than its high-order counterpart, and is up to 2.5 times more computationally efficient than optimized finite-difference schemes using the standard stencil. Our new optimized finite-difference method provides a powerful tool for large-scale, high-frequency 3D modeling and high-resolution 3D inversion.

\section{Acknowledgments}

This work was supported by U.S. Department of Energy through contract DEAC52-06NA25396 to Los Alamos National Laboratory (LANL). The computation was performed using super-computers of LANL's Institutional Computing Program. We thank three anonymous reviewers for their valuable comments.

\section{Appendix A. The coefficients of our optimized FD scheme when $2 M=16$}

For 2D modeling:

$$
\begin{aligned}
d_{1,0}= & -8.74634088067635 E-4 \times r^{7}-1.80530560296097 E-3 \times r^{6} \\
& -4.40512972481673 E-4 \times r^{5}+4.740188476633666 E-3 \times r^{4} \\
& -1.930978022543496 E-5 \times r^{3}-2.92328221171893 E-1 \times r^{2} \\
& -6.58101498708345 E-8 \times r+1.25420636437969, \\
d_{2,0}= & 7.93317828964018 E-4 \times r^{7}+1.61433256585486 E-3 \times r^{6} \\
& +3.97244786277123 E-4 \times r^{5}+5.46057645976549 E-3 \times r^{4} \\
& +1.73781972873916 E-5 \times r^{3}+5.88754971188371 E-2 \times r^{2} \\
& +5.91706982879834 E-8 \times r-1.23406473759703 E-1, \\
& -6.50217700538851 E-4 \times r^{7}-1.16449260340413 E-3 \times r^{6} \\
& -3.24403734066325 E-4 \times r^{5}-9.11483710059994 E-3 \times r^{4} \\
& -1.41739982312600 E-5 \times r^{3}+2.33184077551615 E-2 \times r^{2} \\
& -4.82326094707544 E-8 \times r+3.46342451534453 E-2,
\end{aligned}
$$




$$
\begin{aligned}
d_{4,0}= & 4.67529510541428 E-4 \times r^{7}+7.32736676632388 E-4 \times r^{6} \\
& +2.32444388955328 E-4 \times r^{5}+8.464197666855254 E-3 \times r^{4} \\
& +1.01438593426278 E-5 \times r^{3}-3.17586249260511 E-2 \times r^{2} \\
& +3.44988852042879 E-8 \times r-1.19674942518101 E-2,
\end{aligned}
$$

$$
\begin{aligned}
d_{5,0}= & -2.98416281187033 E-4 \times r^{7}-3.99380750669364 E-4 \times r^{6} \\
& -1.48203388388213 E-4 \times r^{5}-6.01788793192501 E-3 \times r^{4} \\
& -6.46543538517443 E-6 \times r^{3}+2.41912754935119 E-2 \times r^{2} \\
& -2.19855171569984 E-8 \times r+4.15554391204146 E-3,
\end{aligned}
$$

$$
\begin{aligned}
d_{6,0}= & 1.67882669698981 E-4 \times r^{7}+1.88195874702691 E-4 \times r^{6} \\
& +8.30579218603960 E-5 \times r^{5}+3.48461963201376 E-3 \times r^{4} \\
& +3.61873162287129 E-6 \times r^{3}-1.49875789940005 E-2 \times r^{2} \\
& +1.22979142197165 E-8 \times r-1.29213888778954 E-3,
\end{aligned}
$$

$$
\begin{aligned}
d_{7,0}= & -6.22209937489143 E-5 \times r^{7}-6.44890425871692 E-5 \times r^{6} \\
& -3.02936928954918 E-5 \times r^{5}-1.33386143898282 E-3 \times r^{4} \\
& -1.31215186728213 E-6 \times r^{3}+6.70228205200379 E-3 \times r^{2} \\
& -4.44653967516776 E-9 \times r+3.15659916047599 E-4,
\end{aligned}
$$

$$
\begin{aligned}
d_{8,0}= & 6.84740881090240 E-6 \times r^{7}+1.14082245705934 E-5 \times r^{6} \\
& +3.03727593705750 E-6 \times r^{5}+2.36122782444105 E-4 \times r^{4} \\
& +1.26768491232397 E-7 \times r^{3}-1.53347270556276 E-3 \times r^{2} \\
& +4.21617557752767 E-10 \times r-4.51948990428065 E-5,
\end{aligned}
$$




$$
\begin{aligned}
d_{1,1}= & 2.13188763071246 E-6 \times r^{7}-7.41025068776257 E-5 \times r^{6} \\
& +2.31652037371554 E-6 \times r^{5}-2.59495924602038 E-3 \times r^{4} \\
& +1.20637183170338 E-7 \times r^{3}+5.21123771632193 E-2 \times r^{2} \\
& +4.42258843694177 E-10 \times r-4.20967682664542 E-7 .
\end{aligned}
$$

For 3D modeling:

$$
\begin{aligned}
d_{1,0,0}= & .26627215252963 E-3 \times r^{7}-7.91679373564790 E-4 \times r^{6} \\
& +1.08663532410570 E-3 \times r^{5}+2.54974226454794 E-2 \times r^{4} \\
& +3.23083288193913 E-5 \times r^{3}-3.97704676886853 E-1 \times r^{2} \\
& +7.95584310128586 E-8 \times r+1.25425295688331, \\
d_{2,0,0}= & -2.83291379048757 E-3 \times r^{7}+8.52796449228369 E-4 \times r^{6} \\
& -9.45353822586534 E-4 \times r^{5}-8.82015372858580 E-3 \times r^{4} \\
& -2.81364895458027 E-5 \times r^{3}+6.73021045987599 E-2 \times r^{2} \\
& -6.93180036837075 E-8 \times r-1.23448809066664 E-1, \\
& \\
d_{3,0,0}= & 2.32775473203342 E-3 \times r^{7}-5.56793042789852 E-4 \times r^{6} \\
& +7.77649035879584 E-4 \times r^{5}+2.45547234243566 E-3 \times r^{4} \\
& +2.31537892801923 E-5 \times r^{3}+1.61900960524164 E-2 \times r^{2} \\
& +5.70523152308121 E-8 \times r+3.46683979649506 E-2, \\
& -4.14559953526389 E-8 \times r-1.19918511290930 E-2, \\
d_{4,0,0}= & -1.68883462553539 E-3 \times r^{7}+3.03535823592644 E-4 \times r^{6} \\
& -5.64777117315819 E-4 \times r^{5}+2.44582905523866 E-4 \times r^{4} \\
& -1.68215579314751 E-5 \times r^{3}-2.62344345204941 E-2 \times r^{2} \\
&
\end{aligned}
$$




$$
\begin{aligned}
& d_{5,0,0}=1.08994931098070 E-3 \times r^{7}-1.41445142143525 E-4 \times r^{6} \\
& +3.64794490139160 E-4 \times r^{5}-8.86057426195227 E-4 \times r^{4} \\
& +1.08681882832738 E-5 \times r^{3}+2.07238558666603 E-2 \times r^{2} \\
& +2.67876079477806 E-8 \times r+4.17058420250698 E-3, \\
& d_{6,0,0}=-6.39950124405340 E-4 \times r^{7}+6.06079815415080 E-5 \times r^{6} \\
& -2.14633466007892 E-4 \times r^{5}+6.84580412267934 E-4 \times r^{4} \\
& -6.39907927898092 E-6 \times r^{3}-1.29825288653404 E-2 \times r^{2} \\
& -1.57775422151124 E-8 \times r-1.29998325971518 E-3 \text {, } \\
& d_{7,0,0}=2.92716539609611 E-4 \times r^{7}-1.87446062803024 E-5 \times r^{6} \\
& +9.85389372183761 E-5 \times r^{5}-2.40360290348543 E-4 \times r^{4} \\
& +2.94166215515130 E-6 \times r^{3}+5.57066438452790 E-3 \times r^{2} \\
& +7.25741366376659 E-9 \times r+3.18698432679400 E-4, \\
& d_{8,0,0}=-6.42183857909518 E-5 \times r^{7}+3.38552867751042 E-6 \times r^{6} \\
& -2.17377151411164 E-5 \times r^{5}+4.98269067389945 E-5 \times r^{4} \\
& -6.50197868987757 E-7 \times r^{3}-1.19096089679178 E-3 \times r^{2} \\
& -1.60559948991172 E-9 \times r-4.57795411807702 E-5 \text {, } \\
& d_{1,1,0}=-4.47723278782936 E-5 \times r^{7}-7.69502473399932 E-5 \times r^{6} \\
& -1.41765498250133 E-5 \times r^{5}-2.54672045901272 E-3 \times r^{4} \\
& -4.14343385915353 E-7 \times r^{3}+5.00210047924752 E-2 \times r^{2} \\
& -1.01220354410507 E-9 \times r-8.07139347787336 E-8 .
\end{aligned}
$$

\section{References}

[1] P. Moczo, J. O. Robertsson, L. Eisner, The finite-difference time-domain method for modeling of seismic wave propagation, in: V. M. Ru-Shan Wu, R. Dmowska 
(Eds.), Advances in Wave Propagation in Heterogenous Earth, Vol. 48 of Advances in Geophysics, Elsevier, 2007, pp. 421 - 516. doi:10.1016/S00652687(06)48008-0.

URL http://www.sciencedirect.com/science/article/pii/S0065268706480080

[2] M. Dablain, The application of high-order differencing to the scalar wave equation, Geophysics 51 (1) (1986) 54-66. arXiv:http://library.seg.org/doi/pdf/10.1190/1.1442040, doi:10.1190/1.1442040. URL http: //library.seg.org/doi/abs/10.1190/1.1442040

[3] D. Zingg, Comparison of high-accuracy finite-difference methods for linear wave propagation, SIAM Journal on Scientific Computing 22 (2) (2000) 476-502. arXiv:http://epubs.siam.org/doi/pdf/10.1137/S1064827599350320, doi:10.1137/S1064827599350320.

URL http: //epubs . siam.org/doi/abs/10.1137/S1064827599350320

[4] B. Fornberg, Classroom note: Calculation of weights in finite difference formulas, SIAM Review 40 (3) (1998) 685-691. arXiv:http://epubs.siam.org/doi/pdf/10.1137/S0036144596322507, doi:10.1137/S0036144596322507.

URL http://epubs.siam.org/doi/abs/10.1137/S0036144596322507

[5] Y. Liu, M. K. Sen, Scalar wave equation modeling with time-space domain dispersion-relation-based staggered-grid finite-difference schemes, Bulletin of the Seismological Society of America 101 (1) (2011) 141159. arXiv:http://www.bssaonline.org/content/101/1/141.full.pdf+html, doi:10.1785/0120100041.

URL http: //www . bssaonline .org/content/101/1/141 . abstract

[6] J. Etgen, M. OBrien, Computational methods for large-scale 3D acoustic finitedifference modeling: A tutorial, Geophysics 72 (5) (2007) SM223-SM230. arXiv:http://library.seg.org/doi/pdf/10.1190/1.2753753, doi:10.1190/1.2753753. URL http://library.seg.org/doi/abs/10.1190/1.2753753 
[7] B. Fornberg, The pseudospectral method: Comparisons with finite differences for the elastic wave equation, Geophysics 52 (4) (1987) 483-501. arXiv:http://library.seg.org/doi/pdf/10.1190/1.1442319, doi:10.1190/1.1442319. URL http://library.seg.org/doi/abs/10.1190/1.1442319

[8] D. Kosloff, E. Baysal, Forward modeling by a Fourier method, Geophysics 47 (10) (1982) 1402-1412. arXiv:http://library.seg.org/doi/pdf/10.1190/1.1441288, doi:10.1190/1.1441288. URL http://library.seg.org/doi/abs/10.1190/1.1441288

[9] O. Holberg, Computational aspects of the choice of operator and sampling interval for numerical differentiation in large-scale simulation of wave phenomena, Geophysical Prospecting 35 (6) (1987) 629-655. doi:10.1111/j.13652478.1987.tb00841.x.

URL http://dx.doi.org/10.1111/j.1365-2478.1987.tb00841.x

[10] S. K. Lele, Compact finite difference schemes with spectral-like resolution, Journal of Computational Physics 103 (1) (1992) $16-42$. doi:http://dx.doi.org/10.1016/0021-9991(92)90324-R.

URL http://www.sciencedirect.com/science/article/pii/002199919290324R

[11] C. K. Tam, J. C. Webb, Dispersion-relation-preserving finite difference schemes for computational acoustics, Journal of Computational Physics 107 (2) (1993) 262 - 281. doi:10.1006/jcph.1993.1142.

URL http://www.sciencedirect.com/science/article/pii/S0021999183711423

[12] C. Bogey, C. Bailly, A family of low dispersive and low dissipative explicit schemes for flow and noise computations, Journal of Computational Physics 194 (1) (2004) 194 - 214. doi:http://dx.doi.org/10.1016/j.jcp.2003.09.003.

URL http://www.sciencedirect.com/science/article/pii/S0021999103004662

[13] F. Hu, M. Hussaini, J. Manthey, Low-dissipation and low-dispersion Runge-Kutta schemes for computational acoustics, Journal of Computational Physics 124 (1) (1996) 177 - 191. doi:http://dx.doi.org/10.1006/jcph.1996.0052.

URL http://www.sciencedirect.com/science/article/pii/S0021999196900522 
[14] J.-H. Zhang, Z.-X. Yao, Optimized explicit finite-difference schemes for spatial derivatives using maximum norm, Journal of Computational Physics 250 (0) (2013) 511 - 526. doi:http://dx.doi.org/10.1016/j.jcp.2013.04.029.

URL http://www.sciencedirect.com/science/article/pii/S0021999113002945

[15] Z. Haras, S. Ta'asan, Finite difference schemes for long-time integration, Journal of Computational Physics 114 (2) (1994) 265 - 279. doi:http://dx.doi.org/10.1006/jcph.1994.1165.

URL http://www.sciencedirect.com/science/article/pii/S002199918471165X

[16] J. Etgen, A tutorial on optimizing time domain finite-difference schemes: "beyond Holberg", Stanford Exploration Project Report 129 (2007) 33 - 43.

URL http://sepwww. stanford.edu/data/media/public/docs/sep129/john1.pdf

[17] C. Stork, Eliminating nearly all dispersion error from FD modeling and RTM with minimal cost increase, 75th EAGE Conference \& Exhibition incorporating SPE EUROPEC 2013doi:10.3997/2214-4609.20130478.

URL http://earthdoc.eage.org/publication/publicationdetails/?publication=69353

[18] Y. Liu, Globally optimal finite-difference schemes based on least squares, Geophysics 78 (4) (2013) T113-T132. arXiv:http://library.seg.org/doi/pdf/10.1190/geo2012-0480.1, doi:10.1190/geo2012-0480.1.

URL http://library.seg.org/doi/abs/10.1190/geo2012-0480.1

[19] S. Tan, L. Huang, An efficient finite-difference method with high-order accuracy in both time and space domains for modelling scalar-wave propagation, Geophysical Journal Internationaldoi:10.1093/gji/ggu077.

URL http://gji.oxfordjournals .org/content/early/2014/03/26/gji.ggu077

[20] P. D. Lax, B. Wendroff, Difference schemes for hyperbolic equations with high order of accuracy, Communications on Pure and Applied Mathematics 17 (3) (1964) 381-398. doi:10.1002/cpa.3160170311.

URL http://dx.doi.org/10.1002/cpa.3160170311 
[21] J.-B. Chen, A stability formula for Lax-Wendroff methods with fourth-order in time and general-order in space for the scalar wave equation, Geophysics 76 (2) (2011) T37-T42. arXiv:http://geophysics.geoscienceworld.org/content/76/2/T37.full.pdf+html, doi:10.1190/1.3554626.

URL http://geophysics . geoscienceworld .org/content/76/2/T37 . abstract

[22] J. Etgen, S. Gray, Y. Zhang, An overview of depth imaging in exploration geophysics, Geophysics 74 (6) (2009) WCA5-WCA17. arXiv:http://library.seg.org/doi/pdf/10.1190/1.3223188, doi:10.1190/1.3223188.

URL http://library.seg.org/doi/abs/10.1190/1.3223188

[23] J. Virieux, S. Operto, An overview of full-waveform inversion in exploration geophysics, Geophysics 74 (6) (2009) WCC1-WCC26. arXiv:http://library.seg.org/doi/pdf/10.1190/1.3238367, doi:10.1190/1.3238367. URL http://library.seg.org/doi/abs/10.1190/1.3238367

[24] K. Nihei, J. O. A. Robertsson, J. Tromp, J. O. Blanch, Numerical Modeling of Seismic Wave Propagation, Society of Exploration Geophysicists, 2012. arXiv:http://library.seg.org/doi/pdf/10.1190/1.9781560803089, doi:10.1190/1.9781560803089.

URL http://library.seg.org/doi/abs/10.1190/1.9781560803089

[25] R. Clayton, B. Engquist, Absorbing boundary conditions for acoustic and elastic wave equations, Bulletin of the Seismological Society of America 67 (6) (1977) $1529-1540$.

[26] D. Komatitsch, R. Martin, An unsplit convolutional perfectly matched layer improved at grazing incidence for the seismic wave equation, Geophysics 72 (5) (2007) SM155-SM167. arXiv:http://library.seg.org/doi/pdf/10.1190/1.2757586, doi:10.1190/1.2757586.

URL http://library.seg.org/doi/abs/10.1190/1.2757586

[27] Y. Liu, M. Sen, A hybrid scheme for absorbing edge reflections in numerical modeling of wave propagation, Geophysics 75 (2) (2010) A1-A6. 
arXiv:http://library.seg.org/doi/pdf/10.1190/1.3295447, doi:10.1190/1.3295447.

URL http://library.seg.org/doi/abs/10.1190/1.3295447

[28] J. Virieux, SH-wave propagation in heterogeneous media: velocitystress finite-difference method, Geophysics 49 (11) (1984) 1933-1942. doi:10.1190/1.1441605.

URL http://geophysics.geoscienceworld.org/content/49/11/1933. abstract

[29] J. Virieux, P-SV wave propagation in heterogeneous media: velocity-stress finitedifference method, Geophysics 51 (4) (1986) 889-901. doi:10.1190/1.1442147. URL http://geophysics.geoscienceworld.org/content/51/4/889. abstract

[30] A. R. Levander, Fourth-order finite-difference P-SV seismograms, Geophysics 53 (11) (1988) 1425-1436. doi:10.1190/1.1442422.

URL http://geophysics.geoscienceworld.org/content/53/11/1425. abstract

[31] K. Levenberg, A method for the solution of certain non-linear problems in least squares, Quarterly Journal of Applied Mathmatics II (2) (1944) 164-168.

[32] B. Engquist, A. Majda, Absorbing boundary conditions for the numerical simulation of waves, Mathematics of Computation 31 (139) (1977) 629-651.

[33] R. Higdon, Absorbing boundary conditions for elastic waves, Geophysics 56 (2) (1991) 231-241. arXiv:http://dx.doi.org/10.1190/1.1443035, doi:10.1190/1.1443035.

URL http://dx.doi.org/10.1190/1.1443035

[34] J.-P. Berenger, A perfectly matched layer for the absorption of electromagnetic waves, Journal of Computational Physics 114 (2) (1994) 185 - 200. doi:http://dx.doi.org/10.1006/jcph.1994.1159.

URL http://www.sciencedirect.com/science/article/pii/S0021999184711594

[35] J. Krebs, J. Anderson, D. Hinkley, R. Neelamani, S. Lee, A. Baumstein, M. Lacasse, Fast full-wavefield seismic inversion using encoded sources, Geophysics 74 (6) (2009) WCC177-WCC188. 
arXiv:http://library.seg.org/doi/pdf/10.1190/1.3230502, doi:10.1190/1.3230502.

URL http://library.seg.org/doi/abs/10.1190/1.3230502

[36] E. Becache, P. Joly, On the analysis of Berenger's perfectly matched layers for Maxwell's equations, ESAIM: Mathematical Modelling and Numerical Analysis 36 (2002) 87-119. doi:10.1051/m2an:2002004.

[37] S. Abarbanel, H. Qasimov, S. Tsynkov, Long-time performance of unsplit PMLs with explicit second order schemes, Journal of Scientific Computing 41 (1) (2009) 1-12. doi:10.1007/s10915-009-9282-4.

URL http://dx.doi.org/10.1007/s10915-009-9282-4

[38] K. Duru, G. Kreiss, Efficient and stable perfectly matched layer for CEM, Applied Numerical Mathematics 76 (0) (2014) 34-47. doi:http://dx.doi.org/10.1016/j.apnum.2013.09.005.

URL http://www. sciencedirect.com/science/article/pii/S0168927413001347

[39] K. Duru, The role of numerical boundary procedures in the stability of perfectly matched layers, ArXiv e-printsarXiv:1405.0536.

URL http://arxiv.org/abs/1405.0536

[40] J. Zhang, Z. Yao, Optimized finite-difference operator for broadband seismic wave modeling, Geophysics 78 (1) (2013) A13A18. arXiv:http://library.seg.org/doi/pdf/10.1190/geo2012-0277.1, doi:10.1190/geo2012-0277.1.

URL http://library.seg.org/doi/abs/10.1190/geo2012-0277.1

[41] C. Tam, Computational Aeroacoustics: A Wave Number Approach, Cambridge Aerospace Series, Cambridge University Press, 2012.

URL http://books . google.com/books?id=Az5EbI030TkC

[42] R. Snieder, A. Grłt, H. Douma, J. Scales, Coda wave interferometry for estimating nonlinear behavior in seismic velocity, Science 295 (5563) (2002) 2253-2255. arXiv:http://www.sciencemag.org/content/295/5563/2253.full.pdf, 
doi:10.1126/science.1070015.

URL http: //www . sciencemag. org/content/295/5563/2253. abstract

[43] N. Ricker, The form and laws of propagation of seismic wavelets, Geophysics 18 (1) (1953) 10-40. arXiv:http://dx.doi.org/10.1190/1.1437843, doi:10.1190/1.1437843.

URL http://dx.doi.org/10.1190/1.1437843 